\title{
Renormalization of correlations in a barrier billiard: Quadratic irrational trajectories
}

\author{
L.N.C. Adamson* and A.H. Osbaldestin ${ }^{\dagger}$ \\ Department of Mathematics \\ University of Portsmouth \\ Portsmouth \\ PO1 3HF, UK
}

June 20, 2013

\begin{abstract}
We present an analysis of autocorrelation functions in symmetric barrier billiards using a renormalization approach for quadratic irrational trajectories. Depending on the nature of the barrier, this leads to either self-similar or chaotic behaviour. In the self-similar case we give an analysis of the half barrier and present a detailed calculation of the locations, asymptotic heights and signs of the main peaks in the autocorrelation function. Then we consider arbitrary barriers, illustrating that typically these give rise to chaotic correlations of the autocorrelation function which we further represent by showing the invariant sets associated with these correlations. Our main ingredient here is a functional recurrence which has been previously derived and used in work on the Harper equation, strange non-chaotic attractors and a quasi-periodically forced two-level system.

PACS: 05.45.-a, 64.60.Ak
\end{abstract}

Key words: Barrier billiards, renormalization, quadratic irrationals, autocorrelation function

\section{Introduction}

Barrier billiards are a class of dynamical system with autocorrelation functions which have been shown to possess self-similar behaviour. The topic was first motivated by Wiersig [17] who showed that the system could be reduced to a skew-product evolution equation based on the sign of the phase space variable. The key result of Wiersig was that phase space functions for this system exhibit singular continuous spectra. This work also provided evidence that the associated autocorrelation function never decays to 0 or returns to 1.

This result was explored in [1] and it was discovered that for the golden mean frequency, in the case of the so-called half barrier, the autocorrelation function $(\mathrm{ACF})$ has self similar form at Fibonacci times. It was shown that the ACF has peaks of magnitude $1-1 / \sqrt{5}$ at every third Fibonacci number time, whereas it is zero at all other such times. As in previous studies relating to the birth and ACF's of strange nonchaotic attractors ([3], [13], [7], [6]) in addition to analysis of self-similar fluctuations of localized eigenstates of the Harper equation ([5], [11], [9]), key to this analysis is the functional recurrence

$$
Q_{n}(x)=Q_{n-1}(-\omega x) Q_{n-2}\left(\omega^{2} x+\omega\right) .
$$

*email: luke.adamson@port.ac.uk

$\dagger$ email: andrew.osbaldestin@port.ac.uk 
The limitation of this work is that it only deals with the golden mean frequency case, and the purpose of this article is to widen these results to a more general class of quadratic irrational frequencies, namely those of the form

$$
\omega=\frac{\sqrt{m^{2}+4}-m}{2}
$$

These are the numbers whose continued fraction representation is $[m, m, \ldots]$.

The nature of this generalisation is similar to work completed by the second author and his colleague on correlation properties of a quasi-periodically forced two-level system in [10]. This paper built upon the theory previously developed in [4], which focused on the golden mean forcing frequency, and extended the results to quadratic irrationals of this form. The derived functional recurrence in the golden mean case is the additive version of (1.1), and we find that the same is true in the quadratic irrational case. It is shown in [10] that for a particular choice of parameter, this two-level system has an ACF with peaks of magnitude $1-1 / \sqrt{m^{2}+4}$. Somewhat surprisingly it was discovered that there is a dichotomy between the cases $m$ odd, and $m$ even with regard to when these peaks occur.

Similarly, the strong coupling fixed point of the Harper equation and the generalised Harper equation have both been considered for quadratic irrationals of this form ([2], [8]). In [5] while working with the golden mean the authors discover a renormalization strange set which they name "the orchid"; in [8] such a set is produced for each value of $m$ giving rise to a "garden of orchids" (this point will be expanded upon in the conclusion).

In [12] we generalised our study of the barrier billiard problem for the golden mean frequency to arbitrary barriers, and showed that if the height of the barrier is chosen outside the field $\mathbb{Q}(\omega)$ the correlations at successive Fibonacci numbers are chaotic. The chaoticity of the time series of correlations at Fibonacci times was confirmed by the calculation of a positive largest Lyapunov exponent using Rosenstein's method [15]. Furthermore, using the well known embedding theorem of Takens [16] we reconstructed an embedding of the dynamics in three dimensional space, revealing the presence of an invariant set which we may call "the star".

In Section 2 we briefly summarise the equations of motion and derive the functional recurrence which plays the same role as (1.1) for quadratic irrationals. We see that (as expected) it is the multiplicative version of the recurrence found in [10]. Section 3 gives the definition of a map $F$ whose periodic orbits correspond to discontinuities of our renormalized functions $Q_{n}$, and using symbolic dynamics we further deduce information about the period of $Q_{n}$ based on initial discontinuity data. We summarise results from the golden mean frequency case studied in [1] which carry over to our study of quadratic irrational frequencies. In Section 4 we find necessary conditions for $Q_{n}$ to be periodic and give a crucial formula for the discontinuity location sets. In Sections 5 and 6 we extend the work on the half barrier for the golden mean frequency covered in [1] to our class of quadratic irrational frequencies.

It is shown for the half-barrier that peaks of magnitude $1-1 / \sqrt{m^{2}+4}$ occur in the ACF , and that the location of these peaks is determined by the parity of $m$. Furthermore there is a further distinction between the cases of $m \equiv 0(\bmod 4)$ and $m \equiv 2(\bmod 4)$; in the former the ACF only displays positive peaks meaning that there is strict correlation whereas in the latter these peaks alternate in sign which indicates "anticorrelation" which is also seen in the case of $m$ odd.

Finally, in Section 7 we extend our previous work on chaotic correlations in barrier billiards at the golden mean frequency [12] to examples when the frequency is taken from this class of quadratic irrationals. We numerically approximate the autocorrelation function for the system at characteristic times and for each frequency studied we show the existence of invariant sets on which the correlations lie.

\section{Derivation of the relevant equations and functional recurrence}

To begin this section we summarise the derivation of the equations of motion as originally shown in [17].

The problem of symmetric barrier billiards relates to the motion of a point unit mass moving at constant velocity 
in the rectangular chamber $[-1,1] \times[0,1]$. The particle is experiencing elastic collisions with the boundary of this chamber according to the law the angle of incidence is equal to angle of reflection (we ignore trajectories which hit corners). A vertical barrier is placed in the middle of this chamber. It is clear that the evolution of $(|x|, y)$ is simply the integrable evolution of the billiard in $[0,1] \times[0,1]$ which can be written in terms of action-angle variables $\left(\theta_{x}, \theta_{y}\right)$.

To convert to $x-y$ coordinates we define $x=f\left(\theta_{x}\right), y=f\left(\theta_{y}\right)$ where $f$ is the tent map

$$
f(\theta)= \begin{cases}2 \theta, & \theta \in[0,1 / 2] \\ 2(1-\theta), & \theta \in[1 / 2,1] .\end{cases}
$$

Note that $f$ is invertible if we adopt the understanding that $\theta \in[0,1 / 2]$ corresponds to positive velocity.

The nature of the barrier is determined by a barrier function $B(y):[0,1] \rightarrow\{-1,1\}$ which takes the value 1 if the barrier is present at $y$ and -1 elsewhere. We ignore trajectories which hit endpoints of the pieces of the barrier. Figure 1 shows an schematic of the system and its associated barrier function.
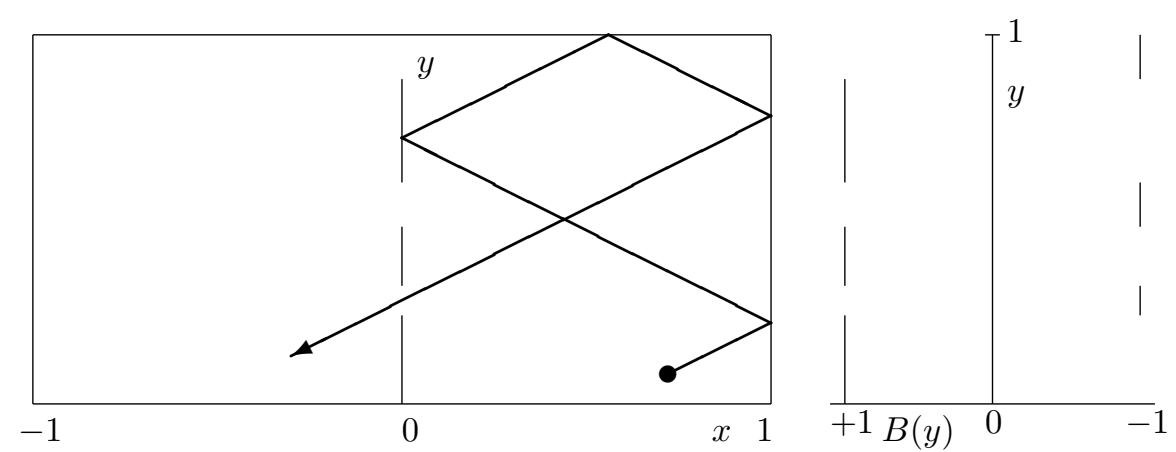

Figure 1: A symmetric barrier billiard and its corresponding barrier function $B(y)$.

Defining

$$
x(t)=s(t)|x(t)|,
$$

where $s(t)$ is the sign of $x$ at time $t$, the question of understanding the system boils down to understanding the evolution of $s$. The sign $s$ can only change sign when $x(t)=0$. Hence taking a stroboscopic sample $x=0$ we repeatedly evaluate $s(t)$ just before the particle strikes the barrier. We define $y_{n}$ be the value of $y$ at time step $n$ (the successive times when $x=0$ ).

If the barrier is present at $y_{n}$ then the sign of $x$ will change, otherwise it will remain the same.

In summary we have reduced the problem of understanding symmetric barrier billiards to that of understanding the skew-product system

$$
\begin{aligned}
& \theta_{n+1}=\theta_{n}+\omega \quad(\bmod 1), \\
& s_{n+1}=\Phi\left(\theta_{n}\right) s_{n} .
\end{aligned}
$$

We see that the solution of the system is given by

$$
\begin{aligned}
& \theta_{n}=\theta_{0}+n \omega \quad(\bmod 1), \\
& s_{n}=s_{0} \prod_{k=0}^{n-1} \Phi\left(\theta_{0}+k \omega\right) .
\end{aligned}
$$

If $\omega$ is rational with then it is clear that $s_{n}$ is periodic and we have a complete understanding of the dynamics. However we can gather no such information if $\omega$ is irrational and so we resort to analysing the autocorrelation 
function $(\mathrm{ACF})$ defined as

$$
C(t)=\left\langle s_{n} s_{n+t}\right\rangle=\lim _{N \rightarrow \infty} \frac{1}{N} \sum_{n=1}^{N} s_{n} s_{n+t} .
$$

Using the same approach as in [3] and [1] we now use the ergodicity in $\theta$ to convert to a phase average:

$$
C(t)=\int_{0}^{1} S_{t}(\theta) d \theta
$$

where

$$
S_{t}(\theta)=\prod_{k=0}^{t-1} \Phi(\theta+k \omega), t \geq 1, \quad S_{0}(\theta)=1 .
$$

Now we restrict our attention to quadratic irrational frequencies of the form.

$$
\omega=\frac{\sqrt{m^{2}+4}-m}{2} .
$$

We define $G_{n}$ to be the "generalised Fibonacci numbers" which satisfy the recursion

$$
G_{n}=m G_{n-1}+G_{n-2}, \quad G_{0}=0, G_{1}=1 .
$$

$\left(G_{n}\right)$ are the numbers whose successive ratios $G_{n} / G_{n+1} \rightarrow \omega$ are the rational convergents to $\omega$ and have the finite continued fraction expansion $[m, m, \ldots, m]$. It is straightforward to verify that

$$
G_{n-1} \omega=G_{n-2}-(-\omega)^{n-1},
$$

We also note the following properties for future reference:

$$
\begin{aligned}
\omega^{-1} & =m+\omega, \\
G_{n} & =\frac{\omega^{-n}}{\sqrt{m^{2}+4}}+O(1) . \\
\omega^{n} & =G_{n+1}-G_{n} \omega .
\end{aligned}
$$

We are interested in the evolution of $S_{t}$ at time $G_{n}$ and so we derive a recurrence relation for $S_{G_{n}}$ as follows:

$$
\begin{aligned}
S_{G_{n}}(\theta) & =\prod_{k=0}^{G_{n}-1} \Phi(\theta+k \omega) \\
& =\prod_{k=0}^{m G_{n-1}-1} \Phi(\theta+k \omega) \prod_{k=m G_{n-1}}^{G_{n}-1} \Phi(\theta+k \omega) \\
& =\left(\prod_{k=0}^{G_{n-1}-1} \Phi(\theta+k \omega) \times \cdots \times \prod_{k=0}^{G_{n-1}-1} \Phi\left(\theta+(m-1) G_{n-1} \omega+k \omega\right)\right) \prod_{k=0}^{G_{n-2}-1} \Phi\left(\theta+m G_{n-1} \omega+k \omega\right) \\
& =\left(\prod_{i=0}^{m-1} S_{G_{n-1}}\left(\theta+i G_{n-1} \omega\right)\right) S_{G_{n-2}}\left(\theta+m G_{n-1} \omega\right) .
\end{aligned}
$$

We now define the re-scaled functions

$$
Q_{n}(x)=S_{G_{n}}\left((-\omega)^{n} x\right),
$$

and using (2.11) we derive the relevant recurrence for $Q_{n}(x)$ :

$$
Q_{n}(x)=\left(\prod_{i=0}^{m-1} Q_{n-1}(-\omega x-i)\right) Q_{n-2}\left(\omega^{2} x+m \omega\right), \quad Q_{0}(x)=1, \quad Q_{1}(x)=\Phi(-\omega x) .
$$




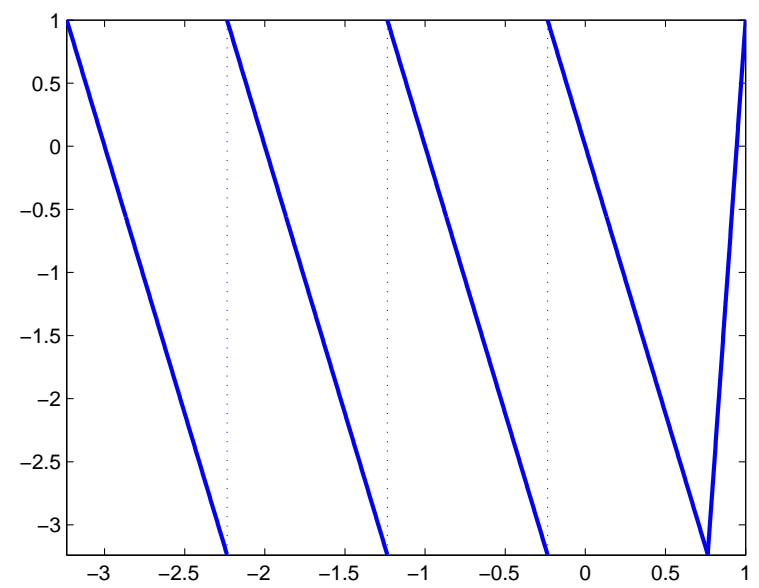

Figure 2: Plot of $F(x)$ for $m=4$

Note that $Q_{1}$ cannot be chosen with arbitrary discontinuity locations as it inherits a symmetry from $\Phi$. This equation is also found in the study of the generalised Harper equation with quadratic irrational flux [8]. As in [1] we can now write

$$
\begin{aligned}
C\left(G_{n}\right) & =\int_{0}^{1} S_{G_{n}}(\theta) \mathrm{d} \theta \\
& =\frac{1}{(-\omega)^{-n}} \int_{0}^{(-\omega)^{-n}} Q_{n}(x) \mathrm{d} x .
\end{aligned}
$$

In order to calculate this we need to consider the global behaviour of $Q_{n}$.

\section{Iterated function system, inverse map $F$ and analysis of the discon- tinuities}

Proceeding in similar fashion to [10], we now define

$$
\begin{aligned}
\phi_{1, i}(x) & =-\omega x-i, \quad i=0,1, \ldots, m-1 \\
\phi_{2, m}(x) & =\omega^{2} x+m \omega .
\end{aligned}
$$

The iterated function system (IFS) defined by the linear contractions $\phi_{1,0}, \phi_{1,1}, \ldots, \phi_{1, m-1}, \phi_{2, m}$ has fundamental interval $I=[-\omega-m+1,1]$, which we can see by noting that $\phi_{1, i}(I)=[-\omega-i,-\omega-i+1]$ and $\phi_{2, m}(I)=[1-\omega, 1]$ so that

$$
\bigcup_{i=0}^{m-1} \phi_{1, i}(I) \cup \phi_{2, m}(I)=I .
$$

It follows that $I$ is the attractor for the IFS. Define (using (2.12))

$$
F(x)= \begin{cases}\phi_{1, i}^{-1}(x)=-(m+\omega) x-(m+\omega) i, & x \in[-\omega-i,-\omega-i+1], i=0,1, \ldots, m-1 ; \\ \phi_{2, m}^{-1}(x)=\left(1+m^{2}+m \omega\right) x-m(m+\omega), & x \in[-\omega+1,1] .\end{cases}
$$

A plot of $F$ in the case $m=4$ is shown in Figure 2. We note that each periodic point of $F$ has $m+1$ possible pre-images, and only one of these is a periodic point on the same orbit. So for any periodic point $y \in I$ we see 
that exactly one of $\phi_{1,0}(y), \ldots, \phi_{1, m-1}(y), \phi_{2, m}(y)$ is also a periodic point of $F$. We ignore the countable set of nonperiodic points whose orbits under $F$ include the points $-\omega-j, j=-1,1, \ldots, m-1$. (These points are eventually periodic.)

Following the approach in [10] we represent the dynamics of $F$ in terms of a code under a point $x \in I$, and we let the symbol $1^{i}$ denote the interval $(-\omega-i,-\omega-i+1)$ and $2^{m}$ denote the interval $(1-\omega, 1]$. The code is then defined to be the sequence $\left(a_{n}^{b_{n}}\right)_{n \geq 0}$ with each $a_{n}^{b_{n}}$ in $\left\{1^{0}, \ldots, 1^{m-1}, 2^{m}\right\}$ where

$$
a_{n}^{b_{n}}= \begin{cases}1^{i}, & F^{n}(x) \in(-\omega-i,-\omega-i+1) \\ 2^{m}, & F^{n}(x) \in(-\omega+1,1] .\end{cases}
$$

This is a crucial definition which will be used throughout Section 5. Our focus for the remainder of the next few sections now turns to barriers leading to periodic behaviour of $Q_{n}$. That is until Section 7, when we study more general barriers. Periodic orbits comprise of periodic codes in $\left\{1^{0}, \ldots, 1^{m-1}, 2^{m}\right\}$ under the shift map

$$
\sigma\left(a_{0}^{b_{0}} a_{1}^{b_{1}} a_{2}^{b_{2}} \ldots\right)=a_{1}^{b_{1}} a_{2}^{b_{2}} \ldots
$$

Due to the expanding nature of $F$, every periodic orbit $y_{0}, \ldots, y_{k-1}$ is uniquely represented by a periodic code $\overline{a_{0}^{b_{0}} a_{1}^{b_{1}} \ldots a_{k-1}^{b_{k-1}}}$ (we will omit the over-line from now on). Given such a code we can calculate the corresponding periodic orbit for $F$. We are looking for solutions of $\phi_{a_{k-1}, b_{k-1}}^{-1} \circ \ldots \circ \phi_{a_{0}, b_{0}}^{-1}(y)=y$, or by taking the inverse they are the solutions of $\phi_{a_{0}, b_{0}} \circ \ldots \circ \phi_{a_{k-1}, b_{k-1}}(y)=y$.

It is straightforward to prove by induction ([10]) that the solution to this equation is given by

$$
y=\frac{-\sum_{j=0}^{k-1} b_{j}(-\omega)^{-1+\sum_{i=0}^{j} a_{i}}}{1-(-\omega)^{\sum a_{j}}} .
$$

Other points on the periodic orbit can be obtained by permuting the code of the orbit.

We define a discontinuity function $R_{n}(x)$ which takes the value -1 if $Q_{n}$ has a discontinuity at $x$ and +1 elsewhere. Formally we define

$$
R_{n}(x)=\frac{Q_{n}(x+)}{Q_{n}(x-)}
$$

Note that as $Q_{n}(x)$ has only finitely many discontinuities on any compact interval we see that $R_{n}(x)$ is well defined. From this definition we see that

$$
\begin{aligned}
R_{n}(x) & =\frac{Q_{n}(x+)}{Q_{n}(x-)} \\
& =\frac{\left(\prod_{i=0}^{m-1} Q_{n-1}\left(\phi_{1, i}(x)-\right)\right) Q_{n-2}\left(\phi_{2}(x)+\right)}{\left(\prod_{i=0}^{m-1} Q_{n-1}\left(\phi_{1, i}(x)+\right)\right) Q_{n-2}\left(\phi_{2}(x)-\right)} \\
& =\left(\prod_{i=0}^{m-1} R_{n-1}\left(\phi_{1, i}(x)\right)\right) R_{n-2}\left(\phi_{2}(x)\right)
\end{aligned}
$$

So $R_{n}$ follows the same recurrence as $Q_{n}$. It is clear that if $Q_{n}$ has period $p$, then $R_{n}$ must have period $m$ where $m \mid p$. In light of what follows in Subsection 3.1 we only consider behaviour on the fundamental interval $I=[-\omega-(m-1), 1]$. For use in the upcoming proof of Proposition 1 we define $D$ as the set of all discontinuities of $Q_{n}$ for every $n$. Formally we write

$$
D=\left\{x \in I: R_{n}(x)=-1 \text { for some } n \geq 0\right\} .
$$

The following proposition and proof are similar to the corresponding result derived for a quasi-periodically forced two-level system in [10], although in this case we work with the multiplicative version of this recurrence. Although the proof is similar, we include it for completeness.

Proposition 1. Let $Q_{n}$ be a periodic orbit of (2.18) and $D$ be defined as in (3.12). Then $D$ consists of a finite collection of periodic orbits of the map $F$. 
Proof. Firstly, suppose $y \in D$. Then $R_{n}(y)=-1$ for some $n \geq 0$. Hence from (3.11) we see that $R_{n-i_{1}}\left(\phi_{i_{1}, j_{1}}(y)\right)=$ -1 for some $i_{1} \in\{1,2\}, j_{1} \in\{0, \ldots, a\}$. Hence $\phi_{i_{1}, j_{1}}(y) \in D$. Continuing in this fashion we deduce that there is a sequence of pairs $\left(i_{1}, j_{1}\right),\left(i_{2}, j_{2}\right), \ldots$ such that $\sigma_{i_{k}, j_{k}} \circ \ldots \circ \phi_{i_{1}, j_{1}}(y) \in D$ for every $k \in \mathbb{N}$. Since $D$ is finite there exist $r, r^{\prime} \in \mathbb{N}$ with $r>r^{\prime}$ and $\sigma_{i_{r^{\prime}} j_{r^{\prime}}} \circ \ldots \circ \phi_{i_{1}, j_{1}}(y)=\sigma_{i_{r}, j_{r}} \circ \ldots \circ \phi_{i_{1}, j_{1}}(y)$. Applying $F^{r}$ to this equation gives $F^{r-r^{\prime}}(y)=y$ so that $y$ is a periodic point of $F$ of period $j$ dividing $r-r^{\prime}$.

Now we let $y_{0}, \ldots, y_{k-1}$ be a periodic orbit of $F$ with $y_{i+1}(\bmod k)=F\left(y_{i}\right)$ for $i=0, \ldots, k-1$ and let $a_{0}^{b_{0}} a_{1}^{b_{1}} \ldots a_{k-1}^{b_{k-1}}$ be the code of this orbit. We have

$$
\phi_{a_{i}, b_{i}}^{-1}\left(y_{i}\right)=y_{i+1} \text { or, equivalently, } \phi_{a_{i-1}, b_{i-1}}\left(y_{i}\right)=y_{i-1} .
$$

As previously discussed, only one of $\phi_{1,0}\left(y_{i}\right), \ldots, \phi_{1, m-1}\left(y_{i}\right), \phi_{2, m}\left(y_{i}\right)$ is periodic. Above we have shown that $y \in D$ implies that $y$ is a periodic point of $F$ and so the contrapositive tells us that if $a_{i-1}=1$ then $\phi_{2, m}\left(y_{i}\right) \notin D$ and if $a_{i-1}=2$ then $\phi_{1, j}\left(y_{i}\right) \notin D$. As a consequence of this we can write

$$
R_{n}\left(y_{i}\right)= \begin{cases}R_{n-1}\left(y_{i-1}\right), & a_{i-1}=1 \\ R_{n-2}\left(y_{i-1}\right), & a_{i-1}=2\end{cases}
$$

Or, more compactly, we can write

$$
R_{n}\left(y_{i}\right)=R_{n-a_{i-1}}\left(y_{i-1}\right) .
$$

From this we see that $R_{n+a_{0}+\ldots+a_{i-1}}\left(y_{i}\right)=R_{n}\left(y_{0}\right)$. Now if $y_{0} \in D$ then $R_{n}\left(y_{0}\right)=-1$ and so we have that $y_{i} \in D$ for every $i$. Hence not only must every point $y$ in $D$ be a periodic point of $F$, but also every point on the orbit of $y$ also lies in $D$. Thus $D$ consists of complete orbits of $F$.

Note that in (3.15) only one of the factors on the right hand side of (3.11) occurs depending on the code $a_{0}, \ldots, a_{k-1}$. Over the whole orbit $y_{0}, \ldots, y_{k-1}, n$ decreases by

$$
l=\sum_{i=0}^{k-1} a_{i}
$$

or, in particular,

$$
R_{n}\left(y_{i}\right)=R_{n-l}\left(y_{i}\right), \quad i=0, \ldots, k-1 .
$$

It is therefore trivial that $m \mid l$, giving us the following proposition.

Proposition 2. The period $m$ of the discontinuity function $R_{n}$ restricted to a periodic orbit $y_{0}, \ldots, y_{k-1}$ of $F$ divides $l$, the sum of the code over the orbit of $F$.

In [7] it was shown that the periodic orbits of (2.18) for $m=1$ corresponding to a given code has period dividing the code sum $l$. The proof of this statement for general quadratic irrationals (arbitrary $m$ ) follows in near identical manner.

\subsection{Reduction of $Q_{n}$ on $\mathbb{R}$ to the fundamental interval}

Due to the fact that the integrals given in (2.20) have to be evaluated on intervals whose length grows exponentially with $n$, we need to consider the behaviour of $Q_{n}$ not just in the fundamental interval $I=[-\omega-(m-1), 1]$, but on the whole of $\mathbb{R}$. Using the fact that $I$ is the fixed point set for the IFS it follows that the behaviour of the functions is driven by their behaviour in $I$.

The following results follow mutatis mutandis from the corresponding results derived for the golden mean frequency as stated in [7].

Lemma 1. Let $Q_{0}, Q_{1}$ be initial conditions for (2.18) on $\mathbb{R}$ and let $\epsilon>0$ be such that $Q_{0}(x)=Q_{1}(x)=1$ for all $x \in[-\omega-(m-1)-\epsilon, 1+\epsilon]$. Then for each $L>1$, there exists $N>0$ (depending only on $L$ ) such that $Q_{n}(x)=1$ for all $x \in[-L, L]$ and all $n>N$. 
This means that if we take initial conditions which take the value unity inside and just outside the fundamental interval, then the value of $Q_{n}$ will eventually be unity at every point.

Proposition 3. Let $Q_{n}$ be a piecewise-constant periodic orbit of (2.18) with period $p$ on $\mathbb{R}$ with $Q_{n}(1+)=Q_{n}(1)$. Then $Q_{n}$ is periodic with period $p$ on the fundamental interval $I$. Conversely, suppose that $Q_{n}$ is periodic with period $p$ on $I$. Then there exists a unique extension $\tilde{Q}_{n}$ of $Q_{n}$ such that $\tilde{Q}_{n}$ is periodic with period $p$ on $\mathbb{R}$.

In essence we can take this as meaning that $Q_{n}$ is periodic with period $p$ on $I$ iff the unique extension $\tilde{Q}_{n}$ on $\mathbb{R}$ is also periodic with the same period $p$. This is important for us because initially we only have discontinuity data in the fundamental interval, and this tells us that if we can find the periodic extension to $Q_{n}$ on $\mathbb{R}$ then we can understand the global behaviour of these functions. On that note, we have the crucial result.

Proposition 4. Let $Q_{0}, Q_{1}$ be piecewise-constant initial conditions for (2.18) on $\mathbb{R}$ with $Q_{0}(1+)=Q_{0}(1), Q_{1}(1+)=$ $Q_{1}(1)$. Suppose $Q_{n}$ is periodic of period $p$ on the fundamental interval $I$. Then the sequence $Q_{n}$ converges to the unique periodic extension $\tilde{Q}_{n}$ given by Proposition 3, i.e., for all integers $r \geq 0$ we have $Q_{r+n p}(x) \rightarrow \tilde{Q}_{r}(x)$ as $n \rightarrow \infty$.

Hence initial data on the fundamental interval which results in periodic behaviour uniquely determines an asymptotic (right continuous at 1) global periodic behaviour as required.

\section{Discontinuity locations for periodic orbits}

Letting $L_{n}$ denote the set of discontinuities of $Q_{n}(x)$ we immediately see that $L_{n}$ satisfies the recurrence

$$
L_{n}=\left(\bigcup_{i \in I} \phi_{1, i}^{-1}\left(L_{n-1}\right)\right) \cup \phi_{2}^{-1}\left(L_{n-2}\right) .
$$

As stated in Subsection 3.1, the global discontinuities of $Q_{n}$ are generated from those inside the fundamental interval, which are themselves elements of period orbits of the map $F$ as seen in Proposition 1. Equation (3.7) shows that such periodic orbits are composed of elements of the field $\mathbb{Q}(\omega)=\{a+b \omega: a, b \in \mathbb{Q}\}$. Thus $L_{n}$ consists of members of $\mathbb{Q}(\omega)$, because the maps $\phi_{1, j}^{-1}, \phi_{2, m}^{-1}$ act on $\mathbb{Q}(\omega)$ as

$$
\begin{aligned}
\phi_{1, j}^{-1}(a+b \omega) & =-(m(a+j)+b)-(a+j) \omega, \\
\phi_{2, m}^{-1}(a+b \omega) & =\left(\left(1+m^{2}\right) a-m^{2}+m b\right)+(m a+b-m) \omega .
\end{aligned}
$$

Rather than consider a globally periodic orbit, we consider an orbit asymptotic to it generated from discontinuity

data on the fundamental interval. By the results of Subsection 3.1 this orbit is eventually periodic and identical to the one we desire on any bounded subset of $\mathbb{R}$.

With the notation that for $x \in \mathbb{R},\lceil x\rceil$ denotes the ceiling of $x$, namely $\min \{n \in \mathbb{Z}: n \geq x\}$, in [10] the following result is established:

Proposition 5. The discontinuity location sets $\left(L_{n}\right)$ arising from applying recurrence (2.18) to initial conditions in which $Q_{0}$ has a single discontinuity at $a+b \omega \in I$, and $Q_{1}$ has a single discontinuity at each of $\phi_{1, j}^{-1}(a+b \omega), j=$ $0, \ldots, m-1$, are $L_{0}=\{a+b \omega\}$ and for $n \geq 1$

$$
L_{n}=c^{(n)}+d^{(n)} \omega+M_{n}
$$

where

$$
M_{n}=\left\{\lceil i \omega\rceil+m i+i \omega: i=l_{n}, \ldots, r_{n}\right\}
$$

with

$$
l_{n}, r_{n}= \begin{cases}-\left(G_{n+1}-G_{n}\right), G_{n}-1, & n \text { odd } \\ -G_{n}, G_{n+1}-G_{n}-1, & n \text { even }\end{cases}
$$


and where

$$
\begin{aligned}
& c^{(n)}=(-1)^{n}\left(G_{n+1} a+G_{n} b\right), \\
& d^{(n)}=(-1)^{n}\left(G_{n} a+G_{n-1} b\right) .
\end{aligned}
$$

\section{Periodic orbits for the half-barrier}

As an example of periodic behaviour in our fundamental recurrence, and thus self-similarity of the autocorrelation function, in this and the next section we confine our attention to the barrier known as the "half-barrier." In $x-y$ coordinates this is the line segment $[0,1 / 2]$ on the $y$-axis $(x=0)$. Note the symmetry $\Phi(\theta)=\Phi(1-\theta)$ in the barrier function, and that $\Phi$ is extended with period one. The function $\Phi(\theta)$ in this case is shown in Figure 3. It is shown in [14] that for irrational trajectories, barriers consisting of a single piece have singular continuous spectra.

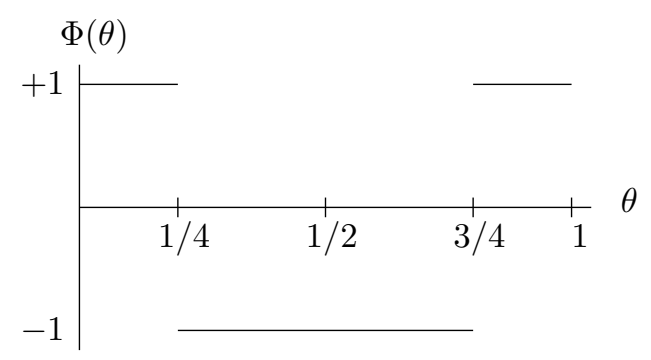

Figure 3: The barrier function $\Phi(\theta)$ for the half-barrier.

An arbitrary barrier $\Phi$ can be decomposed into a product of barriers of this type as shown in [12]. In [17] Wiersig provides numerical results for the ACF of the half barrier for the golden mean frequency, showing that it has peaks at $\simeq 0.55$. Following this it was later proved in [1] that these peaks are of magnitude $1-1 / \sqrt{5}=0.5527 \ldots$ and that they alternate in sign, occurring at every third Fibonacci number.

Following the preparatory work in this section, in the next section we generalise these results and provide a rigorous calculation of the ACF for this particular barrier, showing that the asymptotic magnitude of the peaks is $1-$ $1 / \sqrt{m^{2}+4}$ and that the location of these peaks depends on the parity of $m$. In the case of $m$ even there is a further surprising difference in relation to the signs of these peaks.

\subsection{Relevant periodic orbits and initial conditions}

Recalling the results of Section 2, we see that $Q_{n}$ satisfy the recurrence given in (2.18), and that the initial conditions are given by $Q_{0}(x)=1$ and $Q_{1}(x)=\Phi(-\omega x)$. With $\Phi$ as above the discontinuities of $Q_{1}$ inside the fundamental interval are dependent on $m$ : indeed, a straightforward calculation tells us that if $1 \leq m \leq 3$ then the discontinuities are at $\pm \omega^{-1} / 4$ or, using (2.12), at $\pm(2+\omega) / 4$; whereas for $m \geq 4$ the discontinuities are at $-\omega^{-1} / 4,-3 \omega^{-1} / 4$ or, again using $(2.12),-(2+\omega) / 4,-3(2+\omega) / 4$. We now assume that $m>1$ (the golden mean case $m=1$ having been tackled in [1]), although as we shall later see this assumption is in fact unnecessary because in the case $m \equiv 1$ $(\bmod 4)$, the analysis for $m=1$ and $m \geq 5$ is in fact qualitatively the same. 
5.1.1 $m=2$

When $m=2$ then the orbits of the discontinuities in the fundamental interval are

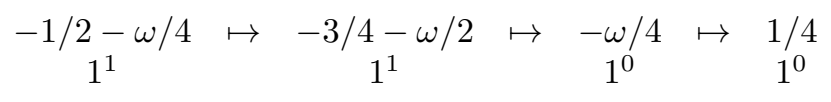

and

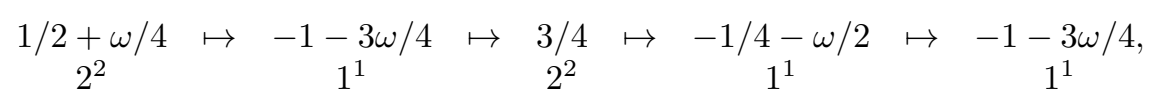

where beneath we indicate the code. We see that $-1 / 2-\omega / 4$ is on the period four orbit $P^{1}=\{-1 / 2-\omega / 4,-3 / 4-$ $\omega / 2,-\omega / 4,1 / 4\}$ with code sum equal to four. Whereas $1 / 2+\omega / 4$ is pre-periodic to the period three orbit $P^{2}=$ $\{-1-3 \omega / 4,3 / 4,-1 / 4-\omega / 2\}$ with code sum also equal to four. Recalling the results of Section 3 this implies the discontinuity locations in the fundamental interval eventually have period a divisor of the code sum four. Table 1 shows where the functions $Q_{n}$ have discontinuities on the fundamental interval, the final column indicates the values of the function at zero. We see that from $Q_{3}$ onwards we have periodic repetition of the discontinuities, the first cycle of which we have indicated with the double lines.

\begin{tabular}{|c|c|c|c|}
\hline$Q_{0}$ & - & - & +1 \\
\hline$Q_{1}$ & $1 / 2+\omega / 4$ & $-1 / 2-\omega / 4$ & +1 \\
\hline$Q_{2}$ & - & $-3 / 4-\omega / 2$ & -1 \\
\hline \hline$Q_{3}$ & $-1-3 \omega / 4$ & $-\omega / 4$ & +1 \\
\hline$Q_{4}$ & $3 / 4$ & $1 / 4$ & +1 \\
\hline$Q_{5}$ & - & $-1 / 2-\omega / 4$ & +1 \\
\hline$Q_{6}$ & $-1 / 4-\omega / 2$ & $-3 / 4-\omega / 2$ & -1 \\
\hline \hline$Q_{7}$ & $-1-3 \omega / 4$ & $-\omega / 4$ & +1 \\
\hline$Q_{8}$ & $3 / 4$ & $1 / 4$ & +1 \\
\hline
\end{tabular}

Table 1: Discontinuities in the fundamental interval for $m=2$

The resulting periodic orbit is shown in Figure 4. Similar pictures for higher values of $m$ can be generated and we generally find that the plot of $Q_{n}$ either looks like the examples from the first column of this figure where the function is predominantly +1 or -1 (from which we would deduce that the ACF is non-zero), or they look like the plots in the second column where the values of the functions take the values \pm 1 in fairly equal measure (from which we would suspect that the ACF is zero). We will put these observations on firmer footing in Section 6 .

\subsection{2 $m=3$}

When $m=3$ the orbits are

$$
\begin{array}{ccccc}
-3 / 4-\omega / 4 & \mapsto & -1 / 2-\omega / 4 & \mapsto & -5 / 4-\omega / 2 \\
1^{1} & & 1^{1} & & 1^{2} \\
& & & & \\
-7 / 4-3 \omega / 4 & \mapsto & -\omega / 4 & \mapsto & 1 / 4 \\
1^{2} & & 1^{0} & & 1^{0}
\end{array}
$$

and

$$
\begin{array}{ccccccc}
3 / 4+\omega / 4 & \mapsto & -3 / 4-\omega / 2 & \mapsto & -1 / 4-\omega / 4 & \mapsto & -2-3 \omega / 4 \\
2^{3} & & 1^{1} & & 1^{1} & & 1^{2} \\
\mapsto & 3 / 4 & \mapsto & -3 / 2-3 \omega / 4 & \mapsto & -3 / 4-\omega / 2 . \\
& 2^{3} & & 1^{2} & & 1^{1}
\end{array}
$$



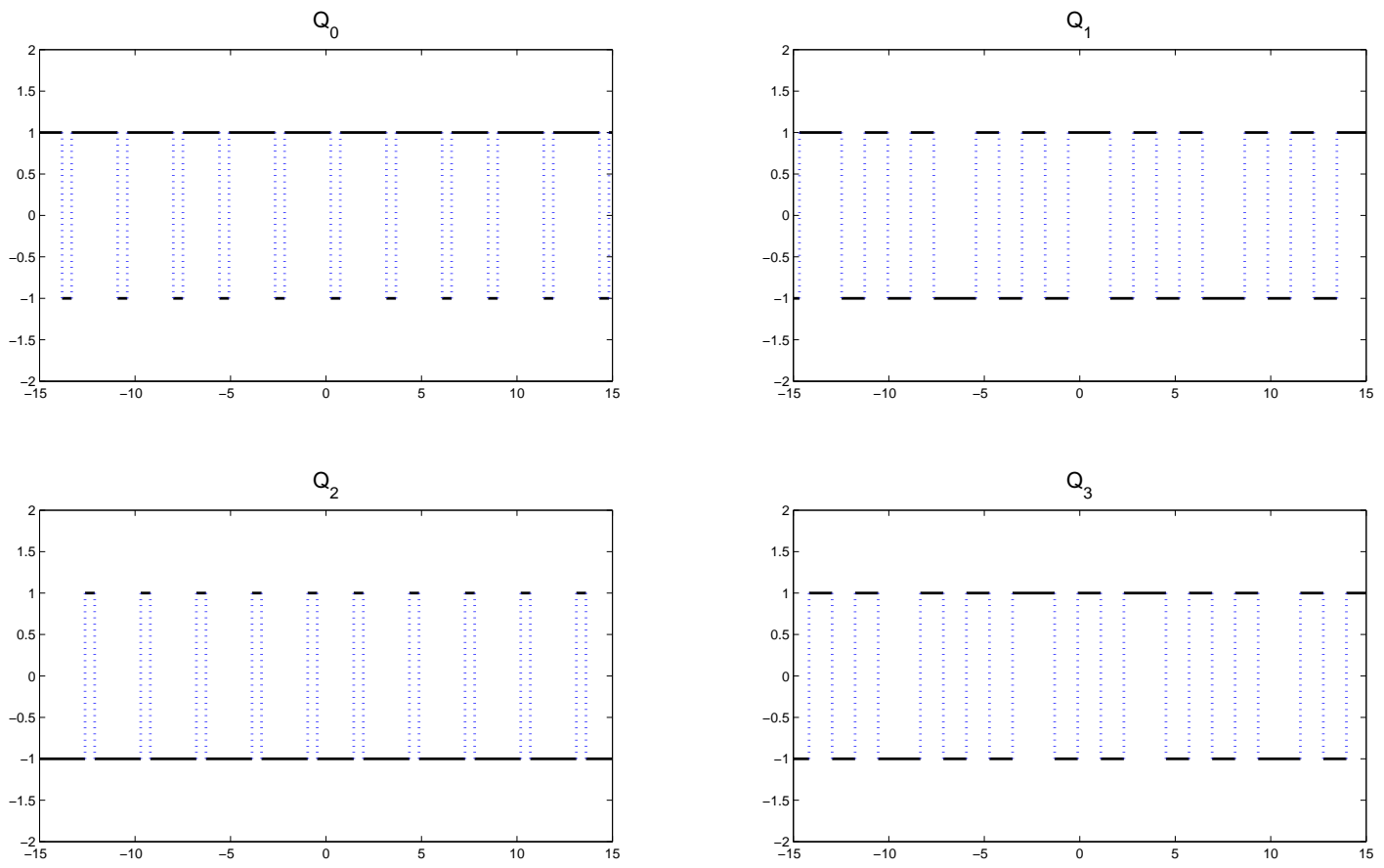

Figure 4: Period 4 orbit for the case $m=2$

In this case we see that $-3 / 4-\omega / 4$ is on the period five orbit $P^{1}=\{-3 / 4-\omega / 4,-1 / 2-\omega / 4,-5 / 4-\omega / 2,-7 / 4-$ $3 \omega / 4,-\omega / 4,1 / 4\}$ with code code sum equal to six. $3 / 4+\omega / 4$ is pre-periodic to the period five orbit $P^{2}=\{-3 / 4-$ $\omega / 2,-1 / 4-\omega / 4,-2-3 \omega / 4,3 / 4,-3 / 2-3 \omega / 4\}$ with code sum also equal to six. The discontinuity locations in the fundamental interval thus have period a divisor of six. A table showing these locations in addition to the values of the functions $Q_{n}$ at zero in the final column is presented in Table 2. The first cycle of the discontinuities is highlighted using the double lines.

\begin{tabular}{|c|c|c|c|}
\hline$Q_{0}$ & - & - & +1 \\
\hline$Q_{1}$ & $3 / 4+\omega / 4$ & $-3 / 4-\omega / 4$ & +1 \\
\hline$Q_{2}$ & - & $-1 / 2-\omega / 4$ & +1 \\
\hline \hline$Q_{3}$ & $-3 / 4-\omega / 2$ & $-5 / 4-\omega / 2$ & -1 \\
\hline$Q_{4}$ & $-1 / 4-\omega / 4$ & $-7 / 4-3 \omega / 4$ & +1 \\
\hline$Q_{5}$ & $-2-3 \omega / 4$ & $-\omega / 4$ & +1 \\
\hline$Q_{6}$ & $3 / 4$ & $1 / 4$ & +1 \\
\hline$Q_{7}$ & - & $-3 / 4-\omega / 4$ & +1 \\
\hline$Q_{8}$ & $-3 / 2-3 \omega / 4$ & $-1 / 2-\omega / 4$ & +1 \\
\hline \hline$Q_{9}$ & $-3 / 4-\omega / 2$ & $-5 / 4-\omega / 2$ & -1 \\
\hline$Q_{10}$ & $-1 / 4-\omega / 4$ & $-7 / 4-3 \omega / 4$ & +1 \\
\hline
\end{tabular}

Table 2: Discontinuities in the fundamental interval for $m=3$

\subsection{3 $m \geq 4$}

When $m \geq 4$ we find that to uniquely determine the code of an orbit we must consider $m$ modulo four. We thus have four cases, and each case will yield a different code for the two periodic orbits corresponding to the two 
discontinuities.

$m \equiv 0(\bmod 4):$

When $m \equiv 0(\bmod 4)$ then the orbits of these two discontinuities are

$$
\begin{array}{ccc}
-m / 4-\omega / 4 & \mapsto & 1 / 4 \\
1^{m / 4} & & 1^{0}
\end{array}
$$

and

$$
\begin{array}{ccc}
-3 m / 4-3 \omega / 4 & \mapsto \quad 3 / 4, \\
1^{3 m / 4} & & 1^{0}
\end{array}
$$

where beneath the points we indicate the code. Hence $-m / 4-\omega / 4$ is on the period two orbit $P^{1}=\{-m / 4-\omega / 4,1 / 4\}$ and $-3 m / 4-3 \omega / 4$ is on the period two orbit $P^{2}=\{-3 m / 4-3 \omega / 4\}$. We see that the code sum is equal to two in both cases, thus implying that the discontinuity locations eventually have period a divisor of two. The functions $Q_{n}$ have discontinuities on the fundamental interval as shown in the Table 3. We have included for reference the value of $Q_{n}$ at zero in the final column. As in the previous table the double lines indicate the first cycle.

\begin{tabular}{|c|c|c|c|}
\hline$Q_{0}$ & - & - & +1 \\
\hline \hline$Q_{1}$ & $-m / 4-\omega / 4$ & $-3 m / 4-3 \omega / 4$ & +1 \\
\hline$Q_{2}$ & $1 / 4$ & $3 / 4$ & +1 \\
\hline \hline$Q_{3}$ & $-m / 4-\omega / 4$ & $-3 m / 4-3 \omega / 4$ & +1 \\
\hline$Q_{4}$ & $1 / 4$ & $3 / 4$ & +1 \\
\hline
\end{tabular}

Table 3: Discontinuities in the fundamental interval for $m \equiv 0(\bmod 4)$

We see the periodicity with period two from $Q_{1}$ onwards. To apply results from previous sections we must choose initial conditions on the fundamental interval that are periodic. We choose $Q_{0}$ to be the function with discontinuities at $1 / 4$ and $3 / 4$ and value +1 at zero, and $Q_{1}$ the function with discontinuities at $-m / 4-\omega / 4$ and $-3 m / 4-3 \omega / 4$ only and value +1 at zero. According to Subsection 3.1 this is enough to determine the globally periodic orbit of (2.18) for the case $m \equiv 0(\bmod 4)$.

$m \equiv 1(\bmod 4):$

If $m \equiv 1(\bmod 4)$ then the orbits are

$$
\begin{array}{ccccc}
-m / 4-\omega / 4 & \mapsto & (1 / 4-3 m / 4)-3 \omega / 4 & \mapsto & (3 / 4-m / 2)-\omega / 2 \\
1^{(m+3) / 4} & & 1^{(3 m+1) / 4} & & 1^{(m-1) / 2} \\
(1 / 2-m / 4)-\omega / 4 & \mapsto & (1 / 4-m / 4)-\omega / 4 & \mapsto & 1 / 4 \\
1^{(m-1) / 4} & & 1^{(m-1) / 4} & & 1^{0}
\end{array}
$$

and

$$
\begin{array}{ccccc}
-3 m / 4-3 \omega / 4 & \mapsto & (3 / 4-m / 4)-\omega / 4 & \mapsto & (1 / 4-m / 2)-\omega / 2 \\
1^{(3 m+1) / 4} & & 1^{(m-1) / 4} & \mapsto \\
(1 / 2-3 m / 4)-3 \omega / 4 & \mapsto & (3 / 4-3 m / 4)-3 \omega / 4 & \mapsto & \\
1^{(3 m+1) / 4} & & 1^{3(m-1) / 4} & 3 / 4 . \\
\end{array}
$$

Hence $-m / 4-\omega / 4$ is on the period six orbit $P^{1}=\{-m / 4-\omega / 4,(1 / 4-3 m / 4)-3 \omega / 4,(3 / 4-m / 2)-\omega / 2,(1 / 2-$ $m / 4)-\omega / 4,(1 / 4-m / 4)-\omega / 4,1 / 4\}$ and $-3 m / 4-3 \omega / 4$ is on the period six orbit $P^{2}=\{-3 m / 4-3 \omega / 4,(3 / 4-$ $m / 4)-\omega / 4,(1 / 4-m / 2)-\omega / 2,(1 / 2-3 m / 4)-3 \omega / 4,(3 / 4-3 m / 4)-3 \omega / 4,3 / 4\}$. In this case the code in both cases sums to six, implying that the discontinuity locations eventually have period a divisor of six. As before we note discontinuity locations in the fundamental interval for $Q_{n}$ in addition to the value at zero in Table 4 . 


\begin{tabular}{|l|c|c|c|}
\hline$Q_{0}$ & - & - & +1 \\
\hline \hline$Q_{1}$ & $-m / 4-\omega / 4$ & $-3 m / 4-3 \omega / 4$ & +1 \\
\hline$Q_{2}$ & $(1 / 4-3 m / 4)-3 \omega / 4$ & $(3 / 4-m / 4)-\omega / 4$ & +1 \\
\hline$Q_{3}$ & $(3 / 4-m / 2)-\omega / 2$ & $(1 / 4-m / 2)-\omega / 2$ & -1 \\
\hline$Q_{4}$ & $(1 / 2-m / 4)-\omega / 4$ & $(1 / 2-3 m / 4)-3 \omega / 4$ & +1 \\
\hline$Q_{5}$ & $(1 / 4-m / 4)-\omega / 4$ & $(3 / 4-3 m / 4)-3 \omega / 4$ & +1 \\
\hline$Q_{6}$ & $1 / 4$ & $3 / 4$ & +1 \\
\hline \hline$Q_{7}$ & $-m / 4-\omega / 4$ & $-3 m / 4-3 \omega / 4$ & +1 \\
\hline$Q_{8}$ & $(1 / 4-3 m / 4)-3 \omega / 4$ & $(3 / 4-m / 4)-\omega / 4$ & +1 \\
\hline$Q_{9}$ & $(3 / 4-m / 2)-\omega / 2$ & $(1 / 4-m / 2)-\omega / 2$ & -1 \\
\hline
\end{tabular}

Table 4: Discontinuities in the fundamental interval for $m \equiv 1(\bmod 4)$

As before we see periodicity after $Q_{1}$ and because we require periodic initial conditions for convenience we choose $Q_{0}$ and $Q_{1}$ as in the previous case.

$m \equiv 2(\bmod 4):$

If $m \equiv 2(\bmod 4)$ the orbits are

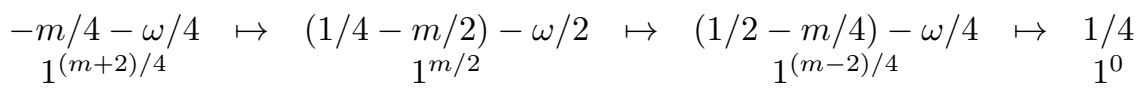

and

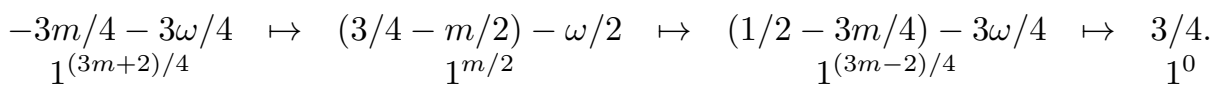

So $-m / 4-\omega / 4$ is on the period four orbit $P^{1}=\{-m / 4-\omega / 4,(1 / 4-m / 2)-\omega / 2,(1 / 2-m / 4)-\omega / 4,1 / 4\}$ and $-3 m / 4-3 \omega / 4$ is on the period four orbit $P^{2}=\{-3 m / 4-3 \omega / 4,(3 / 4-m / 2)-\omega / 2,(1 / 2-3 m / 4)-3 \omega / 4,3 / 4\}$. The code sums to four in both cases, and so the discontinuity locations eventually have period a divisor of four. Table 5 shows discontinuity locations in the fundamental interval for $Q_{n}$ in addition to values at zero, and the rows between the double lines indicate the first cycle of the discontinuities. We see period four repetition from $Q_{1}$ onwards, and

\begin{tabular}{|c|c|c|c|}
\hline$Q_{0}$ & - & - & +1 \\
\hline \hline$Q_{1}$ & $-m / 4-\omega / 4$ & $-3 m / 4-3 \omega / 4$ & +1 \\
\hline$Q_{2}$ & $(1 / 4-m / 2)-\omega / 2$ & $(3 / 4-m / 2)-\omega / 2$ & -1 \\
\hline$Q_{3}$ & $(1 / 2-m / 4)-\omega / 4$ & $(1 / 2-3 m / 4)-3 \omega / 4$ & +1 \\
\hline$Q_{4}$ & $1 / 4$ & $3 / 4$ & +1 \\
\hline \hline$Q_{5}$ & $-m / 4-\omega / 4$ & $-3 m / 4-3 \omega / 4$ & +1 \\
\hline$Q_{6}$ & $(1 / 4-m / 2)-\omega / 2$ & $(3 / 4-m / 2)-\omega / 2$ & -1 \\
\hline
\end{tabular}

Table 5: Discontinuities in the fundamental interval for $m \equiv 2(\bmod 4)$

for simplicity we choose $Q_{0}, Q_{1}$ as in the previous two cases.

$\underline{m \equiv 3(\bmod 4):}$

If $m \equiv 3(\bmod 4)$ then the orbits are given by

$$
\begin{array}{ccccc}
-m / 4-\omega / 4 & \mapsto & (1 / 4-m / 4)-\omega / 4 & \mapsto & (1 / 4-m / 2)-\omega / 2 \\
1^{(m+1) / 4} & & 1^{(m+1) / 4} & & 1^{(m+1) / 2} \\
(1 / 2-3 m / 4)-3 \omega / 4 & \mapsto & (3 / 4-m / 4)-\omega / 4 & \mapsto & 1 / 4 \\
1^{(3 m-1) / 4} & & 1^{(m-3) / 4} & & 1^{0}
\end{array}
$$


and

$$
\begin{array}{ccccc}
-3 m / 4-3 \omega / 4 & \mapsto & (3 / 4-3 m / 4)-3 \omega / 4 & \mapsto & (3 / 4-m / 2)-\omega / 2 \\
1^{3(m+1) / 4} & & 1^{(3 m-1) / 4} & \mapsto & 1^{(m-1) / 2} \\
(1 / 2-m / 4)-\omega / 4 & \mapsto & (1 / 4-3 m / 4)-3 \omega / 4 & \mapsto & 3 / 4 . \\
1^{(m+1) / 4} & & 1^{(3 m-1) / 4} & & 1^{0}
\end{array}
$$

So we have that $-m / 4-\omega / 4$ lies on the period six $P^{1}=\{-m / 4-\omega / 4,(1 / 4-m / 4)-\omega / 4,(1 / 4-m / 2)-\omega / 2,(1 / 2-$ $3 m / 4)-3 \omega / 4,(3 / 4-m / 4)-\omega / 4,1 / 4\}$ and $-3 m / 4-3 \omega / 4$ lies on the period six orbit $P^{2}=\{-3 m / 4-3 \omega / 4,(3 / 4-$ $3 m / 4)-3 \omega / 4,(3 / 4-m / 2)-\omega / 2,(1 / 2-m / 4)-\omega / 4,(1 / 4-3 m / 4)-3 \omega / 4,3 / 4\}$. As in the $m \equiv 1(\bmod 4)$ case both codes sum to six and so the discontinuity locations eventually have period a divisor of six. Table 6 shows discontinuity locations of $Q_{n}$ in the fundamental interval and the values of $Q_{n}$ at zero.

\begin{tabular}{|l|c|c|c|}
\hline$Q_{0}$ & - & - & +1 \\
\hline \hline$Q_{1}$ & $-m / 4-\omega / 4$ & $-3 m / 4-3 \omega / 4$ & +1 \\
\hline$Q_{2}$ & $(1 / 4-m / 4)-\omega / 4$ & $(3 / 4-3 m / 4)-3 \omega / 4$ & +1 \\
\hline$Q_{3}$ & $(1 / 4-m / 2)-\omega / 2$ & $(3 / 4-m / 2)-\omega / 2$ & -1 \\
\hline$Q_{4}$ & $(1 / 2-3 m / 4)-3 \omega / 4$ & $(1 / 2-m / 4)-\omega / 4$ & +1 \\
\hline$Q_{5}$ & $(3 / 4-m / 4)-\omega / 4$ & $(1 / 4-3 m / 4)-3 \omega / 4$ & +1 \\
\hline$Q_{6}$ & $1 / 4$ & $3 / 4$ & +1 \\
\hline \hline$Q_{7}$ & $-m / 4-\omega / 4$ & $-3 m / 4-3 \omega / 4$ & +1 \\
\hline$Q_{8}$ & $(1 / 4-m / 4)-\omega / 4$ & $(3 / 4-3 m / 4)-3 \omega / 4$ & +1 \\
\hline$Q_{9}$ & $(1 / 4-m / 2)-\omega / 2$ & $(3 / 4-m / 2)-\omega / 2$ & -1 \\
\hline
\end{tabular}

Table 6: Discontinuities in the fundamental interval for $m \equiv 3(\bmod 4)$

The discontinuities repeat with period six from $Q_{1}$ onwards, and so we choose $Q_{0}$ and $Q_{1}$ as in the previous three cases.

An important note: Comparing Table 1 with Table 5 and Table 2 with Table 6 (in addition to examining the corresponding table in [1] for $m=1$ to Table 4), we see that we can pick the same initial $Q_{0}$ for $m<4$ as we do for $m \geq 4$. Also note the values of $Q_{n}$ at zero are in both cases identical in these tables for $m>1$. In the following calculation of $C\left(G_{n}\right)$ this means that we only have to consider $m$ modulo four, and not treat the cases $m=2$, $m=3$, or indeed $m=1$, separately.

\section{Calculation of the Autocorrelation Function for the half-barrier}

To calculate the ACF we need to know the locations of the discontinuities of $Q_{n}$ on the whole of $\mathbb{R}$. To do this we need to establish global discontinuity location sets which are the limit of $L_{n}$ as $n \rightarrow \infty$. In light of this we define what is meant by the limit of a sequence of sets, as stated in [10]:

Definition. We say $L=\{f(i): i \in \mathbb{Z}\}$ is the limit of a sequence of sets $L_{n}=\left\{f_{n}(i): i \in I_{n}\right\}\left(L_{n} \rightarrow L\right)$, where $I_{n}$ is a finite sequence of consecutive integers, if there exist integer sequences $\left(l_{n}\right),\left(r_{n}\right)$ such that $L \cap L_{n}=\{f(i): i=$ $\left.l_{n}, \ldots, r_{n}\right\}$ with $l_{n} \rightarrow-\infty, r_{n} \rightarrow \infty$ as $n \rightarrow \infty$.

In addition to this definition we state a proposition that was proven in [1]:

Proposition 6. Let $\eta \in \mathbb{R} \backslash \mathbb{Z}$ and let $\left(W_{n}\right)$ be a monotonic increasing sequence satisfying $W_{n} \rightarrow 1$. Define $T_{n}=\left\{i \in \mathbb{Z}:\{i \omega+\eta\} \leq W_{n}\right\}$. Then, for sufficiently large $n, T_{n}$ contains a set $\left\{l_{n}, l_{n}+1, \ldots, 0, \ldots, r_{n}-1, r_{n}\right\}$ with $l_{n} \in\{0,-1,-2, \ldots\}, r_{n} \in\{0,1,2, \ldots\}$ and $r_{n} \rightarrow \infty, l_{n} \rightarrow-\infty$ as $n \rightarrow \infty$. 
The significance of this is that if we have an expression of the form $\{i \omega+\eta\} \leq W_{n}$ as given in the proposition, then we know that the range of $i$ for which it holds tends to the set of all integers as $n \rightarrow \infty$. Thus by Definition 6 we have that there exists a limit set for which the condition holds as $n \rightarrow \infty$. Formally, defining $\tilde{T}_{n}=\left\{\{i \omega+\eta\}: i \in I_{n}\right.$ $\left(I_{n}\right.$ as given in Definition 6$)$ we have $\tilde{T}_{n} \rightarrow \tilde{T}$ where $\tilde{T}=\{\{i \omega+\eta\}: i \in \mathbb{Z}\}$.

In what follows the we will adopt the notation that $L_{n, 1}$ is the set of discontinuities of $Q_{n}$ generated from $P^{1}$ and $L_{n, 2}$ is the set of discontinuities of $Q_{n}$ generated by $P^{2}$. Clearly $L_{n, 1} \cup L_{n, 2}=L_{n}$, where $L_{n}$ is the set containing all the discontinuities of $Q_{n}$.

\section{1 $m \equiv 0(\bmod 4)$}

For $P^{1}=\{1 / 4,-m / 4-\omega / 4\}$ we define the sets

$$
\begin{aligned}
& L^{1,0}=\{\lceil i \omega-1 / 4\rceil+m i+1 / 4+i \omega: i \in \mathbb{Z}\}, \\
& L^{1,1}=\{\lceil(i-1 / 4) \omega\rceil+m(i-1 / 4)+(i-1 / 4) \omega: i \in \mathbb{Z}\} .
\end{aligned}
$$

For $P^{2}=\{3 / 4,-3 m / 4-3 \omega / 4\}$ we define the sets

$$
\begin{aligned}
& L^{2,0}=\{\lceil i \omega-3 / 4\rceil+m i+3 / 4+i \omega: i \in \mathbb{Z}\}, \\
& L^{2,1}=\{\lceil(i-3 / 4) \omega\rceil+m(i-3 / 4)+(i-3 / 4) \omega: i \in \mathbb{Z}\} .
\end{aligned}
$$

Proposition 7. The sets defined above satisfy

$$
\begin{aligned}
& L_{n, 1} \rightarrow L^{1, i} \quad n=i \quad \bmod 2, \\
& L_{n, 2} \rightarrow L^{2, i} \quad n=i \quad \bmod 2,
\end{aligned}
$$

where $i=0,1$.

Proof. To begin we note the trivial fact that $n \equiv i(\bmod 2)$ implies that $G_{n} \equiv i(\bmod 4)$.

For $P^{1}$ we will only prove the result for $n \equiv 1(\bmod 2)$, the case $n \equiv 0(\bmod 2)$ being almost identical to the case in [1]. Proposition 5 tells us that, $n$ being odd, we have

$$
L_{n, 1}=\left\{-G_{n+1} / 4+\lceil i \omega\rceil+m i+\left(i-G_{n} / 4\right) \omega: i=-\left(G_{n+1}-G_{n}\right), \ldots, G_{n}-1\right\} .
$$

Replacing $i$ by $i+\left(G_{n}-1\right) / 4$ (noting that $\left.\left(G_{n}-1\right) / 4, G_{n+1} / 4 \in \mathbb{Z}\right)$ we see that

$$
\begin{aligned}
L_{n, 1} & =\left\{-G_{n+1} / 4+\left\lceil\left(i+\left(G_{n}-1\right) / 4\right) \omega\right\rceil+\left(i+\left(G_{n}-1\right) / 4\right) m+(1-1 / 4) \omega: i=-\left(G_{n+1}-\left(3 G_{n}+1\right) / 4\right), \ldots, 3\left(G_{n}-1\right) / 4\right\} \\
& =\left\{\left\lceil i \omega-\left(G_{n+1}-m G_{n}-\omega G_{n}+\omega\right) / 4\right\rceil+m(i-1 / 4)+(i-1 / 4) \omega: i=-\left(G_{n+1}-\left(3 G_{n}+1\right) / 4\right), \ldots, 3\left(G_{n}-1\right) / 4\right\} .
\end{aligned}
$$

Now, using the fact that $G_{n+1}-m G_{n}=G_{n-1}$ and $G_{n-1}-\omega G_{n}=\omega^{n}$ we need to show that

$$
\left\lceil(i-1 / 4) \omega-\omega^{n} / 4\right\rceil=\lceil(i-1 / 4) \omega\rceil,
$$

for a suitable range of $i$. For this to be true we need $\left\lceil(i-1 / 4) \omega-\omega^{n} / 4\right\rceil \geq(i-1 / 4) \omega$, or, equivalently,

$$
1+(i-1 / 4) \omega-\omega^{n} / 4-\left\lceil(i-1 / 4) \omega-\omega^{n} / 4\right\rceil=\left\{(i-1 / 4) \omega-\omega^{n} / 4\right\} \leq 1-\omega^{n} / 4,
$$

recalling that $\{x\}=1-(\lceil x\rceil-x), \forall x \in \mathbb{R} \backslash \mathbb{Z}$. Hence (6.8) has the form suitable for application of Proposition 6. Hence (6.7) holds for a suitable range of $i$ as required.

The proof of the results for $P^{2}$ follow in similar fashion.

Now that we have identified the two sets of discontinuities we need to see how they interlace in order to calculate $C\left(G_{n}\right)$. We will let $L_{i}^{a, n}$ denote the $i^{t h}$ member of the set $L^{a, n}, a=1,2$. Using Proposition 5 we see that

$$
L_{i+1}^{a, n}-L_{i}^{a, n}=(m \text { or }(m+1))+\omega .
$$

Equation (6.9) is a relation which will be used throughout the rest of this section as it is true for every case of $m$ modulo four. 


\subsection{1 $n \equiv 0(\bmod 2)$}

When $n \equiv 0(\bmod 2)$, from $(6.1)$ and $(6.3)$ we see that

$$
\begin{aligned}
L_{i}^{2,0}-L_{i}^{1,0} & =(\lceil i \omega-3 / 4\rceil+m i+3 / 4+i \omega)-(\lceil i \omega-1 / 4\rceil+m i+1 / 4+i \omega) \\
& =\lceil i \omega-3 / 4\rceil-\lceil i \omega-1 / 4\rceil+1 / 2 \\
& = \pm 1 / 2 .
\end{aligned}
$$

Assuming $L_{i}^{2,0}-L_{i}^{1,0}=1 / 2$ and using (6.9), we have the possible configurations shown in Figure 5 . These configurations are clearly similar and qualitatively the same when $L_{i}^{2,0}-L_{i}^{1,0}=-1 / 2$. There cannot be adjacent intervals of length $1 / 2$, because in that case $L_{i+1}^{1,0}-L_{i}^{1,0}$ or $L_{i+1}^{2,0}-L_{i}^{2,0}=1$, which contradicts (6.9). Note that because $Q_{0}$ has discontinuities only at $1 / 4$ and $3 / 4$ and value +1 at zero, it has an interval of length $1 / 2$ in which its value is -1 . Hence the value on all intervals of length $1 / 2$ is -1 (see Figure 3). Similar to the argument in [1], we
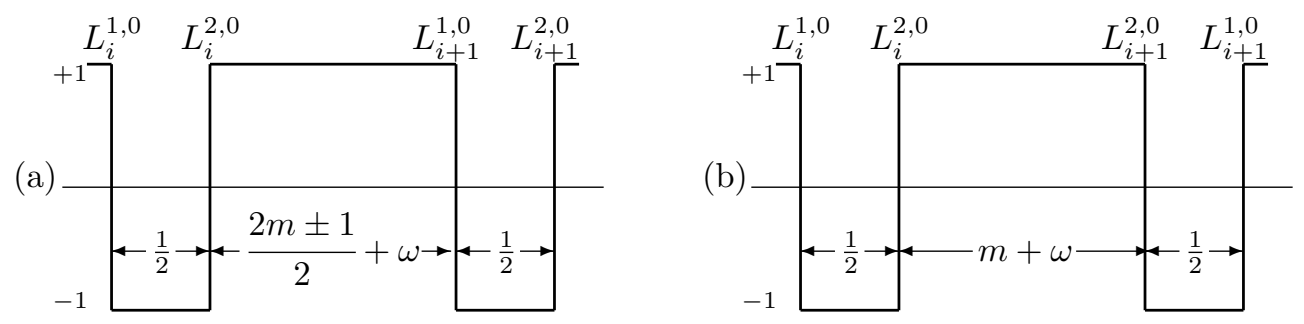

Figure 5: Allowed configurations for the interlacing of $L^{1,0}$ and $L^{2,0}$

see that the graph of $Q_{n}$ consists of intervals of length $1 / 2$ followed by intervals of length $((2 m-1) / 2$ or $(2 m+1) / 2$ or $m)+\omega$ with value +1 . Each of the intervals of length $1 / 2$ has a discontinuity from $L^{1,0}$ associated with it, and on an interval of length $\omega^{-n}=G_{n+1}+\omega G_{n}$ there are $G_{n}+O(1)$ such points as we can see by looking at (6.1). Hence

$$
C\left(G_{n}\right)=\frac{1}{(-\omega)^{-n}} \int_{0}^{(-\omega)^{-n}} Q_{n}(x) \mathrm{d} x=\frac{1}{\omega^{-n}}\left(\omega^{-n}-2 \times \frac{1}{2}\left(G_{n}+O(1)\right)\right) \rightarrow 1-\frac{1}{\sqrt{m^{2}+4}},
$$

using the fact that $G_{n} \omega^{n} \rightarrow 1 / \sqrt{m^{2}+4}$ as $n \rightarrow \infty(2.13)$.

\subsection{2 $n \equiv 1(\bmod 2)$}

In this case using (6.2) and (6.4) we have

$$
\begin{aligned}
L_{i}^{1,1}-L_{i}^{2,1} & =(\lceil(i-1 / 4) \omega\rceil+m(i-1 / 4)+(i-1 / 4) \omega)-(\lceil(i-3 / 4) \omega\rceil+m(i-3 / 4)+(i-3 / 4) \omega) \\
& =\left(\frac{m}{2} \text { or } \frac{m+2}{2}\right)+\frac{\omega}{2} .
\end{aligned}
$$

Similarly we obtain that

$$
L_{i+1}^{2,1}-L_{i}^{1,1}=\left(\frac{m}{2} \text { or } \frac{m+2}{2}\right)+\frac{\omega}{2}
$$

So the two sets are alternating with $L_{i}^{2,1}<L_{i}^{1,1}<L_{i+1}^{2,1}<L_{i+1}^{1,1}<\ldots$

As in [1] we now partition $\mathbb{R}$ into the intervals $\left[L_{i}^{1,1}, L_{i+1}^{1,1}\right]$, and we see the possible configurations in Figure 6 . By the initial conditions $L_{0}^{1,1}=-m / 4-\omega / 4$ and with $Q_{1}(0)=+1$, the value of $Q_{n}$ is +1 from $L_{i}^{1,1}$ to $L_{i+1}^{2,1}$ and -1 from $L_{i+1}^{2,1}$ to $L_{i+1}^{1,1}$ as depicted. 

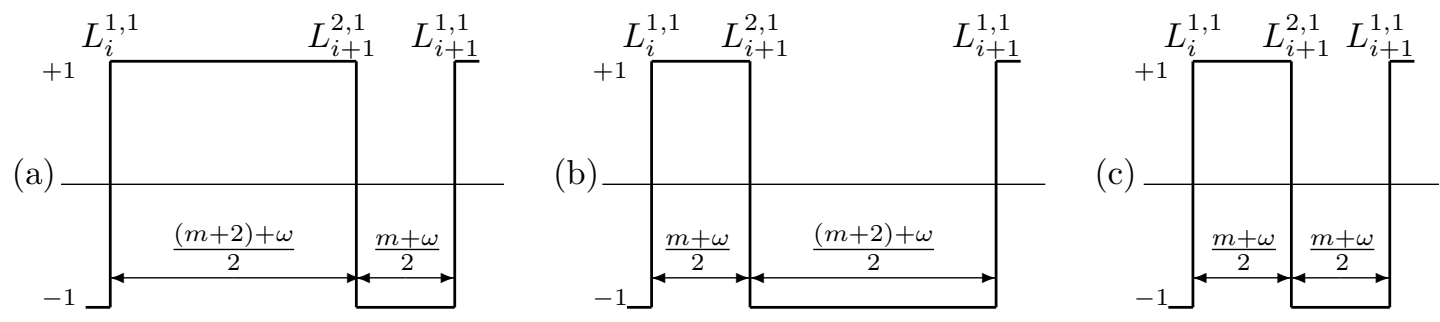

Figure 6: Possibilities for each interval between $L_{i}^{1,1}$ and $L_{i+1}^{1,1}$

We see that the only contribution is from a $(m+1)+\omega$ interval, so suppose $L_{i+1}^{1,1}-L_{i}^{1,1}=(m+1)+\omega$. From (6.2) we see that

$$
L_{i+1}^{1,1}-L_{i}^{1,1}=\lceil(i+3 / 4) \omega\rceil-\lceil(i-1 / 4) \omega\rceil+m+\omega .
$$

Hence a necessary and sufficient condition for $\left[L_{i}^{1,1}, L_{i+1}^{1,1}\right]$ to be a $(m+1)+\omega$ interval is

$$
\lceil(i+3 / 4) \omega\rceil-\lceil(i-1 / 4) \omega\rceil=1 .
$$

Set $r=\lceil(i-1 / 4) \omega\rceil$, and we can the rewrite (6.11) as $r-\omega<(i-1 / 4) \omega<r$ or

$$
r-3 \omega / 4<i \omega<r+\omega / 4,
$$

meaning that $i \omega$ lies in an interval (of length $<1$ ) with centre $r-\omega / 4$. Let $\alpha$ denote the $(m+1)+\omega$ interval shown in Figure $6(\mathrm{a})$, so that $L_{i+1}^{2,1}-L_{i}^{1,1}=(m+2) / 2+\omega / 2$ implies an $\alpha$ interval. Let $\beta$ be the interval shown in Figure 6(b), in which case $L_{i+1}^{2,1}-L_{i}^{1,1}=m / 2+\omega / 2$. From (6.4) and (6.2) we have

$$
L_{i+1}^{2,1}-L_{i}^{1,1}=\lceil(i+1 / 4) \omega\rceil-\lceil(i-1 / 4) \omega\rceil+m / 2+\omega / 2 .
$$

So we have an $\alpha$ interval if $\lceil(i+1 / 4) \omega\rceil-\lceil(i-1 / 4) \omega\rceil=1$ and a $\beta$ interval if $\lceil(i+1 / 4) \omega\rceil-\lceil(i-1 / 4) \omega\rceil=0$.

This means that we have an $\alpha$ interval if

$$
i \omega<r-\omega / 4
$$

and a $\beta$ interval if

$$
i \omega>r-\omega / 4 \text {. }
$$

Hence we can see that the intervals in which $i \omega$ lies corresponding to $\alpha$ and $\beta$ intervals have the same length, and so by the uniform distribution of $i \omega$ we have the same asymptotically the same number of $\alpha$ and $\beta$ intervals and hence we conclude that the value of the integral is zero. In conclusion we have found that

$$
\lim _{l \rightarrow \infty} C\left(G_{2 l+k}\right)= \begin{cases}1-1 / \sqrt{m^{2}+4}, & k=0 ; \\ 0, & k=1 .\end{cases}
$$

The result of this analysis is that the autocorrelation has only positive peaks. A numerical evaluation of the ACF is shown in Figure 7 for $m=4$, and the arrows show the theoretical location of the peaks (in addition to the corresponding value of $t$ ). Indeed, we observe that the entire ACF is predominantly non negative. 


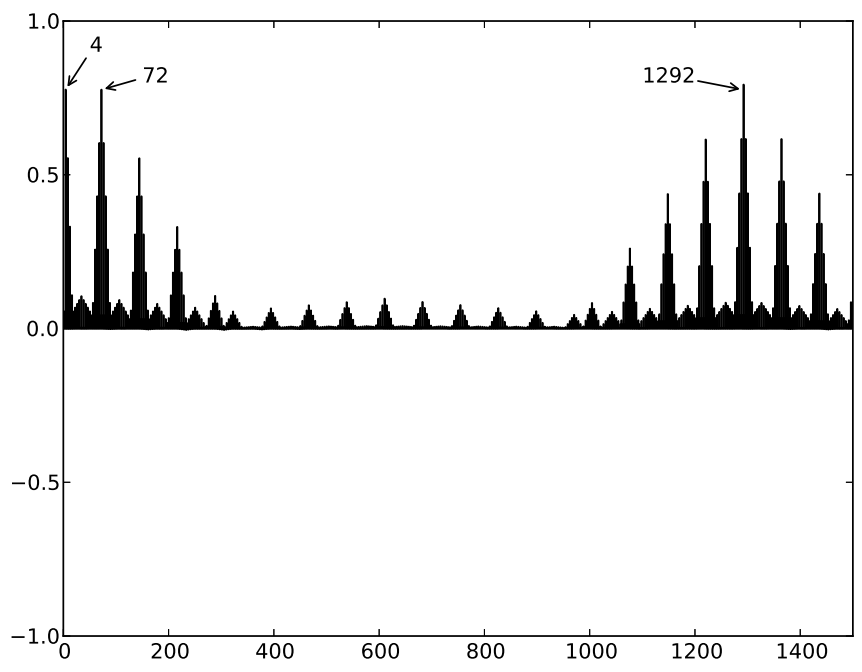

Figure 7: Numerical evaluation of the ACF when $m=4$ for the half barrier (exact theoretical peaks asymptotically of height $1-1 / \sqrt{20}=0.776 \ldots$ are shown by the arrows)

\section{2 $m \equiv 1(\bmod 4)$}

As before we must calculate the global discontinuity location sets for $Q_{n}$ for each of the periodic orbits. For $P^{1}=\{1 / 4,-m / 4-\omega / 4,(1 / 4-3 m / 4)-3 \omega / 4,(3 / 4-m / 2)-\omega / 2,(1 / 2-m / 4)-\omega / 4,(1 / 4-m / 4)-\omega / 4\}$ we define the sets

$$
\begin{aligned}
& L^{1,0}=\{\lceil i \omega-1 / 4\rceil+m i+1 / 4+i \omega: i \in \mathbb{Z}\}, \\
& L^{1,1}=\{\lceil(i-1 / 4) \omega\rceil+m(i-1 / 4)+(i-1 / 4) \omega: i \in \mathbb{Z}\}, \\
& L^{1,2}=\{\lceil(i+1 / 4) \omega+3 / 4\rceil+m(i+1 / 4)-3 / 4+(i+1 / 4) \omega: i \in \mathbb{Z}\}, \\
& L^{1,3}=\{\lceil(i-1 / 2) \omega+1 / 4\rceil+m(i-1 / 2)-1 / 4+(i-1 / 2) \omega: i \in \mathbb{Z}\}, \\
& L^{1,4}=\{\lceil(i-1 / 4) \omega+1 / 2\rceil+m(i-1 / 4)-1 / 2+(i-1 / 4) \omega: i \in \mathbb{Z}\}, \\
& L^{1,5}=\{\lceil(i-1 / 4) \omega-1 / 4\rceil+m(i-1 / 4)+1 / 4+(i-1 / 4) \omega: i \in \mathbb{Z}\} .
\end{aligned}
$$

For $P^{2}=\{3 / 4,-3 m / 4-3 \omega / 4,(3 / 4-m / 4)-\omega / 4,(1 / 4-m / 2)-\omega / 2,(1 / 2-3 m / 4)-3 \omega / 4,(3 / 4-3 m / 4)-3 \omega / 4\}$ the sets are

$$
\begin{aligned}
& L^{2,0}=\{\lceil i \omega+1 / 4\rceil+m i-1 / 4+i \omega: i \in \mathbb{Z}\}, \\
& L^{2,1}=\{\lceil(i-3 / 4) \omega\rceil+m(i-3 / 4)+(i-3 / 4) \omega: i \in \mathbb{Z}\}, \\
& L^{2,2}=\{\lceil(i-1 / 4) \omega-3 / 4\rceil+m(i-1 / 4)+3 / 4+(i-1 / 4) \omega: i \in \mathbb{Z}\}, \\
& L^{2,3}=\{\lceil(i+1 / 2) \omega+3 / 4\rceil+m(i+1 / 2)-3 / 4+(i+1 / 2) \omega: i \in \mathbb{Z}\}, \\
& L^{2,4}=\{\lceil(i+1 / 4) \omega+1 / 2\rceil+m(i+1 / 4)-1 / 2+(i+1 / 4) \omega: i \in \mathbb{Z}\}, \\
& L^{2,5}=\{\lceil(i-3 / 4) \omega-3 / 4\rceil+m(i-3 / 4)+3 / 4+(i-3 / 4) \omega: i \in \mathbb{Z}\} .
\end{aligned}
$$

Note that these sets are identical in the case $m=1$, as we have assumed $Q_{0}$ has exactly the same discontinuities in each case and so by Proposition 5 they must be the same.

Proposition 8. The sets defined above satisfy

$$
\begin{aligned}
& L_{n, 1} \rightarrow L^{1, i} \quad n=i \quad \bmod 6, \\
& L_{n, 2} \rightarrow L^{2, i} \quad n=i \quad \bmod 6,
\end{aligned}
$$


where $i=0,1, \ldots, 5$.

Proof. The following table is useful in completing this proof:

\begin{tabular}{|c|c|}
\hline$n(\bmod 6)$ & $G_{n}(\bmod 4)$ \\
\hline 0 & 0 \\
\hline 1 & 1 \\
\hline 2 & 1 \\
\hline 3 & 2 \\
\hline 4 & 3 \\
\hline 5 & 1 \\
\hline
\end{tabular}

We see that the table is valid for the case $m=1$, which was used to derive these sets in [1] for this value of $m$. These sets for general $m \equiv 1$ modulo four therefore follow in near identical fashion.

\subsection{1 $n \equiv 0(\bmod 3)$}

Due to the fact that $L^{1,0}$ and $L^{2,0}$ are identical to the corresponding sets for the case $m \equiv 0(\bmod 4)$ (see equations (6.1), (6.13) and (6.3), (6.20)), the configurations are as shown in Figure 5 for $n \equiv 0(\bmod 6)$, and the value of $C\left(G_{n}\right)$ is (asymptotically) $1-1 / \sqrt{m^{2}+4}$.

On the other hand for $n \equiv 3(\bmod 6)$, using $(6.16)$ and $(6.22)$ we note that

$$
L_{i+1}^{1,3}-L_{i}^{2,3}= \pm 1 / 2 .
$$

The configurations are hence the same as in Figure 5, but because $Q_{3}(0)=-1$ the graphs are multiplied by minus one. Hence the intervals of length $1 / 2$ are now positive. In light of this we could say that the graph of $Q_{3}$ is qualitatively the same as the graph of $-Q_{0}$. Now because $n$ is odd, we are integrating from right to left, which is the same as integrating from left to right if we multiply by minus one. The factor $(-\omega)^{-n}$ is negative, and so these factors of minus one cancel. Hence the autocorrelation function $C\left(G_{n}\right)$ has asymptotic value equal to minus one times the value obtained for $n \equiv 0(\bmod 6)$, hence asymptotically $C\left(G_{n}\right)$ takes the value $-\left(1-1 / \sqrt{m^{2}+4}\right)$.

\subsection{2 $n \equiv 1(\bmod 3)$}

For $n \equiv 1(\bmod 6)$ note that $L^{1,1}$ and $L^{2,1}$ are identical to the corresponding sets produced in the case $m \equiv 0$ $(\bmod 4)$ for $n \equiv 1(\bmod 2)$. Hence the interlacing of the discontinuities is the same as in Figure 6 , so as in that case the ACF is zero i.e. $C\left(G_{6 k+1}\right)=0$.

On the other hand if $n \equiv 4(\bmod 6)$ then equations $(6.17)$ and $(6.23)$ give

$$
L_{i}^{2,4}-L_{i}^{1,4}=\left(\frac{m}{2} \text { or } \frac{m+2}{2}\right)+\frac{\omega}{2},
$$

and

$$
L_{i+1}^{1,4}-L_{i}^{2,4}=\left(\frac{m}{2} \text { or } \frac{m+2}{2}\right)+\frac{\omega}{2} .
$$

Hence we have that the discontinuities alternate as in the previous case, except that $L_{i}^{2,4}<L_{i+1}^{1,4}<L_{i+1}^{2,4}<\ldots$. So the configurations are identical to those in Figure 6 except for the reordering of the discontinuities. 
The calculation of the ACF is similar to that for the case $n \equiv 1(\bmod 6)$, and we deduce that the asymptotic value of the integral is also zero.

\subsection{3 $n \equiv 2(\bmod 3)$}

Using equations (6.15) and (6.21) we see that

$$
\begin{aligned}
L_{i}^{1,2}-L_{i}^{2,2} & =(\lceil(i+1 / 4) \omega+3 / 4\rceil+m(i+1 / 4)-3 / 4+(i+1 / 4) \omega)-(\lceil(i-1 / 4) \omega-3 / 4\rceil+m(i-1 / 4)+3 / 4+(i-1 / 4) \omega) \\
& =(1 \text { or } 2)+\frac{m}{2}-\frac{3}{2}+\frac{\omega}{2} \\
& =\left(\frac{m-1}{2} \text { or } \frac{m+2}{2}\right)+\frac{\omega}{2}>0,
\end{aligned}
$$

and, similarly,

$$
L_{i+1}^{2,2}-L_{i}^{1,2}=\left(\frac{m-1}{2} \text { or } \frac{m+2}{2}\right)+\frac{\omega}{2}>0 .
$$

Hence we have $L_{i}^{2,2}<L_{i}^{1,2}<L_{i+1}^{2,2}<L_{i+1}^{1,2}<\ldots$ Partitioning $\mathbb{R}$ into the intervals $\left[L_{i}^{1,2}, L_{i+1}^{1,2}\right]$ the allowable configurations are shown in Figure 8. Note that by the initial condition $L_{0}^{1,2}=(3 / 4-m / 4)-\omega / 4$ and this condition along with $Q_{2}(0)=+1$, means that (for $m>1$ ) the value of $Q_{n}$ is +1 from $L_{i}^{1,2}$ to $L_{i+1}^{2,2}$ and -1 from $L_{i+1}^{2,2}$ to $L_{i+1}^{1,2}$ as shown. The only contribution to the integrals comes from $m+\omega$ intervals between $L_{i}^{1,2}$ and $L_{i+1}^{1,2}$.
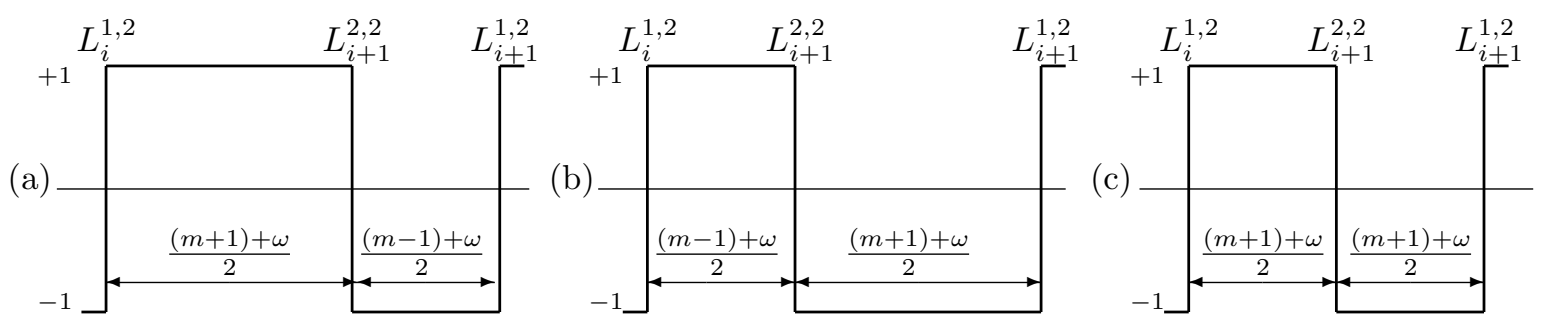

Figure 8: Possibilities for each interval between $L_{i}^{1,1}$ and $L_{i+1}^{1,1}$

So suppose $L_{i+1}^{1,2}-L_{i}^{1,2}=m+\omega$. Using (6.15) we see that

$$
L_{i+1}^{1,2}-L_{i}^{1,2}=\lceil(i+5 / 4) \omega+3 / 4\rceil-\lceil(i+1 / 4) \omega+3 / 4\rceil+m+\omega .
$$

So a necessary and sufficient condition for a $m+\omega$ interval is

$$
\lceil(i+5 / 4) \omega+3 / 4\rceil-\lceil(i+1 / 4) \omega+3 / 4\rceil=0,
$$

or, letting $r=\lceil(i+1 / 4) \omega+3 / 4\rceil$ we can equivalently write (6.2.3) as $r-1<(i+1 / 4) \omega+3 / 4<r-\omega$, or, rearranging we have

$$
r-7 / 4-\omega / 4<i \omega<r-3 / 4-5 \omega / 4 .
$$

Hence $i \omega$ is contained in an interval with centre $r-5 / 4-3 \omega / 4$.

Let $\alpha$ denote the $m+\omega$ shown in Figure 8(a) and $\beta$ denote the $m+\omega$ interval shown in Figure 8(b). We can specify these intervals by noting that if $L_{i+1}^{2,2}-L_{i}^{1,2}=(m-1) / 2+\omega / 2$ we have a $\beta$ interval, and if $L_{i+1}^{2,2}-L_{i}^{1,2}=(m+1) / 2+\omega / 2$ we have a $\alpha$ interval.

Using (6.15) and (6.21) we see that

$$
L_{i+1}^{2,2}-L_{i}^{1,2}=\lceil(i+3 / 4) \omega+1 / 4\rceil-\lceil(i+1 / 4) \omega+3 / 4\rceil+\frac{m}{2}+\frac{1}{2}+\frac{\omega}{2} .
$$


Hence we have a $\beta$ interval if $\lceil(i+3 / 4) \omega+1 / 4\rceil-\lceil(i+1 / 4) \omega+3 / 4\rceil=-1$ and a $\alpha$ interval if $\lceil(i+3 / 4) \omega+1 / 4\rceil-$ $\lceil(i+1 / 4) \omega+3 / 4\rceil=0$.

Using these facts we see that we have a $\beta$ interval if $i \omega<r-3 \omega / 4-5 / 4$ and a $\alpha$ interval if $i \omega>r-3 \omega / 4-5 / 4$. Hence the intervals in which $i \omega$ lies corresponding to $\alpha$ and $\beta$ intervals have equal length, and thus due to the uniform distribution of $i \omega$ we have that the number of $\alpha$ and $\beta$ intervals is the same, and so the asymptotic value of the integral is zero.

Note that for the case $m=1$ the graphs were -1 from $L_{i}^{1,2}$ to $L_{i+1}^{2,2}$, however it is clear that this will not affect the calculation of the autocorrelation function as the net area under the graphs is identical.

In the case $n \equiv 5(\bmod 6)$, using equations (6.18) and (6.24), we deduce that

$$
L_{i}^{1,5}-L_{i}^{2,5}=\left(\frac{m-1}{2} \text { or } \frac{m+2}{2}\right)+\frac{\omega}{2}>0,
$$

and

$$
L_{i+1}^{2,5}-L_{i}^{1,5}=\left(\frac{m-1}{2} \text { or } \frac{m+2}{2}\right)+\frac{\omega}{2}>0 .
$$

Thus we have that the ordering of the discontinuities is $L_{i}^{1,5}<L_{i+1}^{2,5}<L_{i+1}^{1,5}<\ldots$. Due to the distances between them being identical to the those produced in the case $n \equiv 2(\bmod 6)$, and considering the interval $\left[L_{i}^{1,5}, L_{i+1}^{1,5}\right]$ we deduce that the discontinuities interlace in the same way as in the case $n \equiv 2(\bmod 6)$. Using a similar argument as before we therefore deduce that the asymptotic value of the integral of $Q_{n}$ is zero.

Hence in conclusion we have that

$$
\lim _{l \rightarrow \infty} C\left(G_{6 l+3 k}\right)=(-1)^{k}\left(1-\frac{1}{\sqrt{m^{2}+4}}\right)
$$

and

$$
\lim _{l \rightarrow \infty} C\left(G_{6 l+k}\right)=0, \quad k=1,2,4,5 .
$$

Note that this is in agreement with the results obtained for the golden mean $(m=1)$ case studied in [1].

A numerical evaluation of the ACF for $m=5$ demonstrates this behaviour as shown in Figure 9 (theoretical peaks and the corresponding values of $t$ are indicated by the arrows).

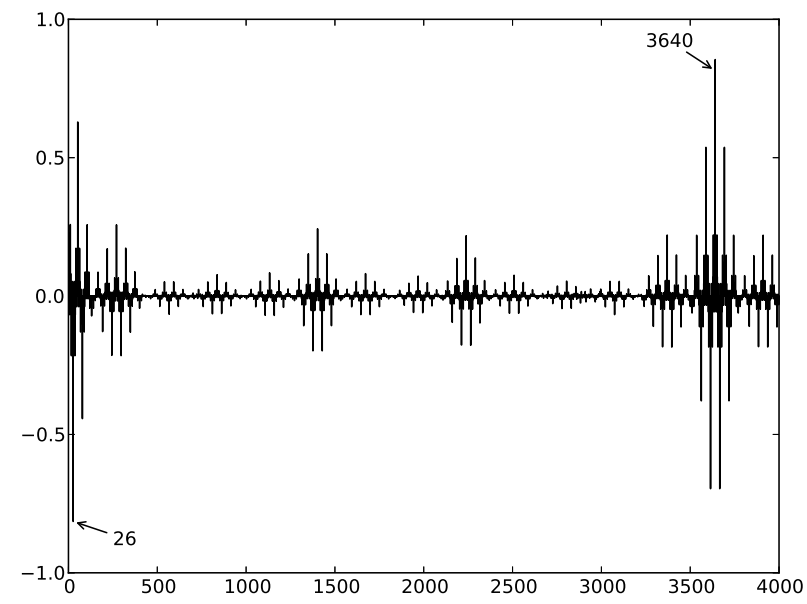

Figure 9: Numerical evaluation of the ACF when $m=5$ for the half barrier (exact theoretical peaks of asymptotic height $\pm(1-1 / \sqrt{29})= \pm 0.814 \ldots$ are shown by the arrows) 


\section{$6.3 m \equiv 2(\bmod 4)$}

Using the same methodology as before we define for the periodic orbit $P^{1}=\{1 / 4,-m / 4-\omega / 4,(1 / 4-m / 2)-$ $\omega / 2,(1 / 2-m / 4)-\omega / 4\}(m \geq 6)$ the sets

$$
\begin{aligned}
& L^{1,0}=\{\lceil i \omega-1 / 4\rceil+m i+1 / 4+i \omega: i \in \mathbb{Z}\}, \\
& L^{1,1}=\{\lceil(i-1 / 4) \omega\rceil+m(i-1 / 4)+(i-1 / 4) \omega: i \in \mathbb{Z}\}, \\
& L^{1,2}=\{\lceil(i+1 / 2) \omega+3 / 4\rceil+m(i+1 / 2)-3 / 4+(i+1 / 2) \omega: i \in \mathbb{Z}\}, \\
& L^{1,3}=\{\lceil(i-1 / 4) \omega-1 / 2\rceil+m(i-1 / 4)+1 / 2+(i-1 / 4) \omega: i \in \mathbb{Z}\},
\end{aligned}
$$

and for $P^{2}=\{3 / 4,-3 m / 4-3 \omega / 4,(3 / 4-m / 2)-\omega / 2,(1 / 2-3 m / 4)-3 \omega / 4\}(m \geq 6)$ we define

$$
\begin{aligned}
& L^{2,0}=\{\lceil i \omega+1 / 4\rceil+m i-1 / 4+i \omega: i \in \mathbb{Z}\}, \\
& L^{2,1}=\{\lceil(i-3 / 4) \omega\rceil+m(i-3 / 4)+(i-3 / 4) \omega: i \in \mathbb{Z}\}, \\
& L^{2,2}=\{\lceil(i+1 / 2) \omega+1 / 4\rceil+m(i+1 / 2)-1 / 4+(i+1 / 2) \omega: i \in \mathbb{Z}\}, \\
& L^{2,3}=\{\lceil(i-3 / 4) \omega-1 / 2\rceil+m(i-3 / 4)+1 / 2+(i-3 / 4) \omega: i \in \mathbb{Z}\}, .
\end{aligned}
$$

Note that these sets are identical in the case $m=2$, as we have assumed $Q_{0}$ has exactly the same discontinuities in each case and so by Proposition 5 they must be the same.

Proposition 9. The sets defined above satisfy

$$
\begin{aligned}
& L_{n, 1} \rightarrow L^{1, i} \quad n=i \quad \bmod 4, \\
& L_{n, 2} \rightarrow L^{2, i} \quad n=i \quad \bmod 4,
\end{aligned}
$$

where $i=0,1,2,3$.

Proof. The following table of $G_{n}$ values modulo 4 will be useful:

\begin{tabular}{|c|c|}
\hline$n(\bmod 4)$ & $G_{n}(\bmod 4)$ \\
\hline 0 & 0 \\
\hline 1 & 1 \\
\hline 2 & 2 \\
\hline 3 & 1 \\
\hline
\end{tabular}

Note that because the first two entries are zero and one, $L^{1,0}$ and $L^{2,0}$ will be identical to the previous two cases. Note also that $L^{1,1}$ and $L^{2,1}$ are identical to previous cases of $m$ modulo four. We will prove the result for $L^{2,2}$, all the other cases follow similarly.

In this case, $n$ being even, we have from Proposition 5 that

$$
L_{n, 2}=\left\{3 G_{n+1} / 4+\lceil i \omega\rceil+m i+\left(i+3 G_{n} / 4\right) \omega: i=-G_{n}, \ldots, G_{n+1}-G_{n}-1\right\} .
$$

Replacing $i$ by $\left(i-3\left(G_{n}-2\right) / 4\right)$ (noting that $\left.\left(G_{n}-2\right) / 4,\left(G_{n+1}-1\right) / 4 \in \mathbb{Z}\right)$ we have

$$
\begin{aligned}
L_{n, 2} & =\left\{3 G_{n+1} / 4+\left\lceil\left(i-3\left(G_{n}-2\right) / 4\right) \omega\right\rceil+\left(i-3\left(G_{n}-2\right) / 4\right) m+(i+3 / 2) \omega: i=-\left(G_{n}+6\right) / 4, \ldots, G_{n+1}-\left(G_{n}+10\right) / 4\right\} \\
& =\left\{\left\lceil i \omega+3\left(G_{n+1}-1-\omega G_{n}+2 \omega-m G_{n}+2 m\right) / 4\right\rceil+m i+3 / 4+(i+3 / 2) \omega: i=-\left(G_{n}+6\right) / 4, \ldots, G_{n+1}-\left(G_{n}+10\right) / 4\right\} \\
& =\left\{\left\lceil i \omega+3\left(\omega^{n}+2 \omega+2(m-2)+3\right) / 4\right\rceil+m i+3 / 4+(i+3 / 2) \omega: i=-\left(G_{n}+6\right) / 4, \ldots, G_{n+1}-\left(G_{n}+10\right) / 4\right\} \\
& =\left\{\left\lceil(i+3 / 2) \omega+9 / 4+3 \omega^{n} / 4\right\rceil+m(i+3 / 2)-9 / 4+(i+3 / 2) \omega: i=-\left(G_{n}+6\right) / 4, \ldots, G_{n+1}-\left(G_{n}+10\right) / 4\right\} \\
& =\left\{\left\lceil(i+1 / 2) \omega+1 / 4+3 \omega^{n} / 4\right\rceil+m(i+1 / 2)-1 / 4+(i+1 / 2) \omega: i=-\left(G_{n}+2\right) / 4, \ldots, G_{n+1}-\left(G_{n}+6\right) / 4\right\}
\end{aligned}
$$


So we need to prove that $\left\lceil(i+1 / 2) \omega+1 / 4+3 \omega^{n} / 4\right\rceil=\lceil(i+1 / 2) \omega+1 / 4\rceil$ for a suitable range of $i$. We can rewrite this condition as $\lceil(i+1 / 2) \omega+1 / 4\rceil \geq(i+1 / 2) \omega+1 / 4+3 \omega^{n} / 4$, or, equivalently

$$
1+(i+1 / 2) \omega+1 / 4-\lceil(i+1 / 2) \omega+1 / 4\rceil=\{(i+1 / 2) \omega+1 / 4\} \leq 1-3 \omega^{n} / 4 .
$$

Hence we have a form suitable for application of Proposition 6. and we conclude that the result is true for a suitable range of $i$ as required.

\subsection{1 $n \equiv 0 \bmod 2$}

For the case $n \equiv 0(\bmod 4)$ we note that due to the fact that $L^{1,0}$ and $L^{2,0}$ are the same as in previous cases, we see that the interlacing of the discontinuities is as in Figure 5 and that the asymptotic value of the autocorrelation function is $1-1 / \sqrt{m^{2}+4}$.

If $n \equiv 2(\bmod 4)$ then using equations $(6.31)$ and $(6.35)$ we have

$$
\begin{aligned}
L_{i}^{1,2}-L_{i}^{2,2} & =(\lceil(i+1 / 2) \omega+3 / 4\rceil+m(i+1 / 2)-3 / 4+(i+1 / 2) \omega-(\lceil(i+1 / 2) \omega+1 / 4\rceil+m(i+1 / 2)-1 / 4+(i+1 / 2) \omega \\
& =(0 \text { or } 1)-1 / 2
\end{aligned}
$$

Note that $L_{-1}^{2,2}=(3 / 4-m / 2)-\omega / 2$ and $L_{-1}^{1,2}=(1 / 4-m / 2)-\omega / 2$, and so because $Q_{2}(0)=-1$ we conclude that the graph of $Q_{n}$ has value +1 on intervals of length $1 / 2$. So the argument is now the same as that produced for $m \equiv 1$ $(\bmod 4)$ in the case $n \equiv 3(\bmod 6)$ and we conclude that the asymptotic value of the integral is $-\left(1-1 / \sqrt{m^{2}+4}\right)$.

\subsection{2 $n \equiv 1(\bmod 2)$}

In the case $n \equiv 1(\bmod 4)$ we note that due to the sets $L^{1,1}$ and $L^{2,1}$ being identical to those in the case $m \equiv 1$ $(\bmod 4)$, that the discontinuities will interlace in exactly the same manner as in Figure 6, and thus we can re-apply the arguments of the previous section to deduce that the asymptotic value of the integral will be zero.

If $n \equiv 3(\bmod 4)$ the using equations $(6.32)$ and $(6.36)$ we see that

$$
\begin{aligned}
L_{i}^{1,3}-L_{i}^{2,3} & =(\lceil(i-1 / 4) \omega-1 / 2\rceil+m(i-1 / 4)+1 / 2+(i-1 / 4) \omega-(\lceil(i-1 / 4) \omega-1 / 2\rceil+m(i-1 / 4)+1 / 2+(i-3 / 4) \omega) \\
& =(0 \text { or } 1)+\frac{m}{2}+\frac{\omega}{2} \\
& =\left(\frac{m}{2} \text { or } \frac{m+2}{2}\right)+\frac{\omega}{2}>0 .
\end{aligned}
$$

Similarly we obtain

$$
L_{i+1}^{2,3}-L_{i}^{1,3}=\left(\frac{m}{2} \text { or } \frac{m+2}{2}\right)+\frac{\omega}{2}>0 .
$$

Hence the discontinuities alternate with $L_{i}^{1,3}<L_{i+1}^{2,3}<L_{i+1}^{1,3}<\ldots$. As a result of this, (with trivial relabelling) the discontinuities interlace as in Figure 6 and using similar arguments as those previously used we deduce that the asymptotic value of the integral is zero.

In summary we conclude that

$$
\lim _{l \rightarrow \infty} C\left(G_{4 l+2 k}\right)=(-1)^{k}\left(1-\frac{1}{\sqrt{m^{2}+4}}\right)
$$

and

$$
\lim _{l \rightarrow \infty} C\left(G_{4 l+k}\right)=0, \quad k=1,3 .
$$

A numerical evaluation of the ACF demonstrating this behaviour in the case $m=2$ is given in Figure 10, with the theoretical peaks shown by the arrows. We remark that in [13] the authors examine the ACF of a strange 
non-chaotic attractor, with driving frequency $\sqrt{2}-1$ (which is our case $m=2$ ) they find numerically that the peaks are of magnitude $\simeq 0.65$. The analysis of the $\mathrm{ACF}$ of a strange non-chaotic attractor will be virtually identical to that for the half barrier case of barrier billiards considered here. Thus the value Pikovsky and Feudel calculated in [13] is in fact $1-1 / \sqrt{8}=0.646 \ldots$

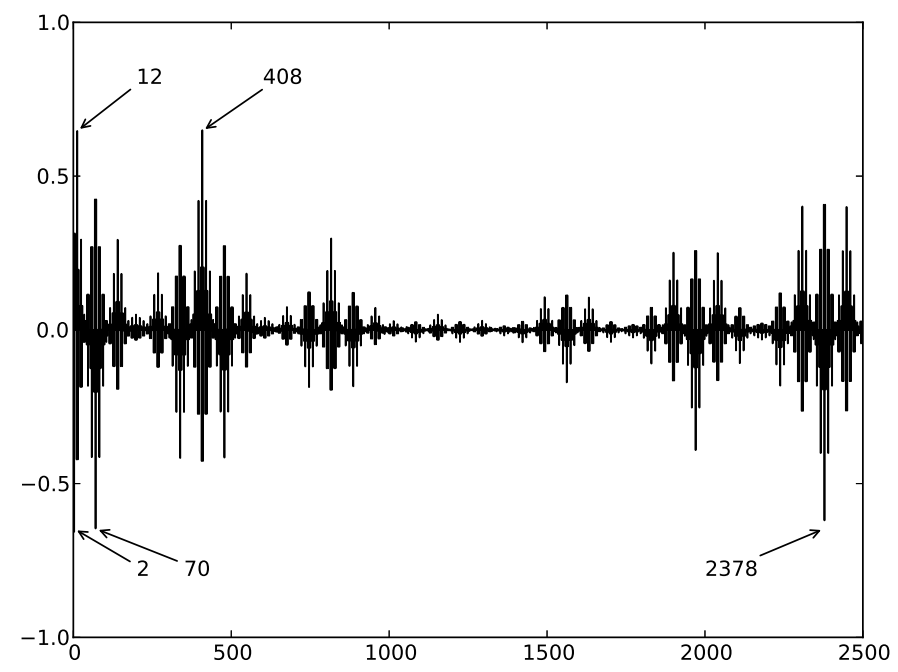

Figure 10: Numerical evaluation of the ACF when $m=2$ for the half barrier (exact theoretical peaks of asymptotic height $\pm(1-1 / \sqrt{8})= \pm 0.646 \ldots$ are shown by the arrows)

\section{4 $m \equiv 3(\bmod 4)$}

For the periodic orbit $P^{1}=\{1 / 4,-m / 4-\omega / 4,(1 / 4-m / 4)-\omega / 4,(1 / 4-m / 2)-\omega / 2,(1 / 2-3 m / 4)-3 \omega / 4,(3 / 4-$ $m / 4)-\omega / 4\}(m \geq 7)$ we define the sets

$$
\begin{aligned}
& L^{1,0}=\{\lceil i \omega-1 / 4\rceil+m i+1 / 4+i \omega: i \in \mathbb{Z}\}, \\
& L^{1,1}=\{\lceil(i-1 / 4) \omega\rceil+m(i-1 / 4)+(i-1 / 4) \omega: i \in \mathbb{Z}\}, \\
& L^{1,2}=\{\lceil(i+3 / 4) \omega+3 / 4\rceil+m(i+3 / 4)-3 / 4+(i+3 / 4) \omega i \in \mathbb{Z}\}, \\
& L^{1,3}=\{\lceil(i-1 / 2) \omega+3 / 4\rceil+m(i-1 / 2)-3 / 4+(i-1 / 2) \omega: i \in \mathbb{Z}\}, \\
& L^{1,4}=\{\lceil(i+1 / 4) \omega+1 / 2\rceil+m(i+1 / 4)-1 / 2+(i+1 / 4) \omega: i \in \mathbb{Z}\}, \\
& L^{1,5}=\{\lceil(i-1 / 4) \omega+1 / 4\rceil+m(i-1 / 4)-1 / 4+(i-1 / 4) \omega: i \in \mathbb{Z}\},
\end{aligned}
$$

and for $P^{2}=\{3 / 4,-3 m / 4-3 \omega / 4,(3 / 4-3 m / 4)-3 \omega / 4,(3 / 4-m / 2)-\omega / 2,(1 / 2-m / 4)-\omega / 4,(1 / 4-3 m / 4)-3 \omega / 4\}$ the sets

$$
\begin{aligned}
& L^{2,0}=\{\lceil i \omega+1 / 4\rceil+m i-1 / 4+i \omega: i \in \mathbb{Z}\}, \\
& L^{2,1}=\{\lceil(i-3 / 4) \omega\rceil+m(i-3 / 4)+(i-3 / 4) \omega: i \in \mathbb{Z}\}, \\
& L^{2,2}=\{\lceil(i+1 / 4) \omega+1 / 4\rceil+m(i+1 / 4)-1 / 4+(i+1 / 4) \omega: i \in \mathbb{Z}\}, \\
& L^{2,3}=\{\lceil(i-1 / 2) \omega+1 / 4\rceil+m(i-1 / 2)-1 / 4+(i-1 / 2) \omega: i \in \mathbb{Z}\}, \\
& L^{2,4}=\{\lceil(i+3 / 4) \omega+1 / 2\rceil+m(i+3 / 4)-1 / 2+(i+3 / 4) \omega: i \in \mathbb{Z}\}, \\
& L^{2,5}=\{\lceil(i-3 / 4) \omega+3 / 4\rceil+m(i-3 / 4)-3 / 4+(i-3 / 4) \omega: i \in \mathbb{Z}\} .
\end{aligned}
$$

These sets are the same when $m=3$ as we have assumed the same initial discontinuity for $Q_{0}$ in each case. This follows from Proposition 5. 
Proposition 10. The sets defined above satisfy

$$
\begin{aligned}
& L_{n, 1} \rightarrow L^{1, i} \quad n=i \quad \bmod 6, \\
& L_{n, 2} \rightarrow L^{2, i} \quad n=i \quad \bmod 6,
\end{aligned}
$$

where $i=0,1, \ldots, 5$.

Proof. The following table is useful for proving these results

\begin{tabular}{|c|c|}
\hline$n(\bmod 6)$ & $G_{n}(\bmod 4)$ \\
\hline 0 & 0 \\
\hline 1 & 1 \\
\hline 2 & 3 \\
\hline 3 & 2 \\
\hline 4 & 1 \\
\hline 5 & 1 \\
\hline
\end{tabular}

The proof for each of these sets follows in identical fashion to the examples already presented earlier.

\subsection{1 $n \equiv 0(\bmod 3)$}

Due to (6.13), (6.41) and (6.19), (6.47) being identical the evaluation of the ACF for $n \equiv 0(\bmod 6)$ is identical to all the previous cases of $m$ modulo four and we conclude that asymptotically the value of $C\left(G_{n}\right)$ is $1-1 / \sqrt{m^{2}+4}$.

If $n \equiv 3(\bmod 6)$ then using equations (6.44) and (6.50) we have that

$$
L_{i}^{1,3}-L_{i}^{2,3}= \pm 1 / 2 .
$$

We also have that $Q_{3}(0)=-1$ and $L_{0}^{1,3}=(1 / 4-m / 2)-\omega / 2$ and $L_{0}^{2,3}=(3 / 4-m / 2)-\omega / 2$, and so the value of $Q_{3}$ between $L_{i}^{1,3}$ and $L_{i}^{2,3}$ must be +1 and so the situation is as in the case $m \equiv 1(\bmod 4), n \equiv 3(\bmod 6)$ and we deduce that the asymptotic value of the integral is $-\left(1-1 / \sqrt{m^{2}+4}\right)$.

\subsection{2 $n \equiv 1(\bmod 3)$}

For $n \equiv 1(\bmod 6)$ we have that $L^{1,1}$ and $L^{2,1}$ are identical to the corresponding sets in the case $m \equiv 1(\bmod 4)$. Hence the interlacing of the inequalities is the same as in Figure 6 and we conclude that the asymptotic value of the integral is zero.

If $n \equiv 4(\bmod 6)$ then using equations $(6.45)$ and $(6.51)$ we have

$$
\begin{aligned}
L_{i}^{2,4}-L_{i}^{1,4} & =L_{i+1}^{1,4}-L_{i}^{2,4} \\
& =\left(\frac{m}{2} \text { or } \frac{m+2}{2}\right)+\frac{\omega}{2}>0 .
\end{aligned}
$$

Hence the configurations are the same as in Figure 6 (with a re-ordering of the discontinuities) and using similar techniques as before we deduce that the integral is zero. 


\subsection{3 $n \equiv 2(\bmod 3)$}

Firstly we consider the case $n \equiv 2(\bmod 6)$, then equations (6.43) and (6.49) tell us that

$$
\begin{aligned}
L_{i}^{1,2}-L_{i}^{2,2} & =L_{i+1}^{2,2}-L_{i}^{1,2} \\
& =\left(\frac{m-1}{2} \text { or } \frac{m+2}{2}\right)+\frac{\omega}{2}>0 .
\end{aligned}
$$

So $L_{i}^{1,2}<L_{i+1}^{2,2}<L_{i+1}^{1,2}<\ldots$

Hence the discontinuities interlace in the same way as they did for $m \equiv 1(\bmod 4)$ in Figure 8. Again it is a similar exercise involving the ceil function to show that the value of the integral is zero.

For the case $n \equiv 5(\bmod 4)$ we reach the same conclusions, the ordering is the same as are the possible configurations and again we conclude that the integral is zero.

So our conclusions for $m \equiv 3(\bmod 4)$ are exactly the same as those for $m \equiv 1(\bmod 4)$. Hence all odd $m$ provide similar behaviour asymptotically.

Figure 11 shows a numerical evaluation of the ACF for $m=3$, and the arrows show the theoretical peaks.

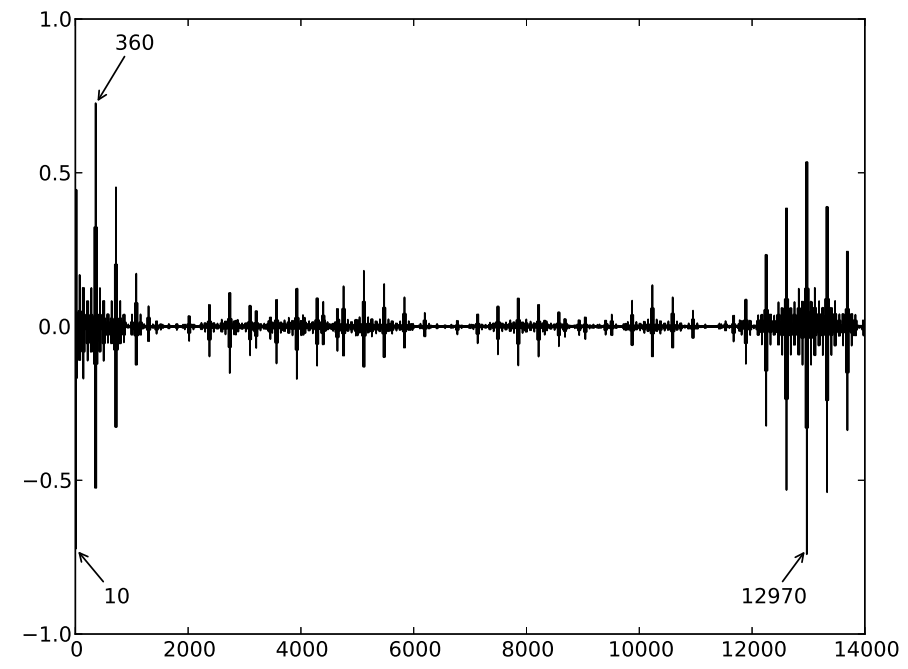

Figure 11: Numerical evaluation of the ACF when $m=3$ for the half barrier (exact theoretical peaks of asymptotic height $\pm(1-1 \sqrt{13})= \pm 0.722 \ldots$ are shown by the arrows)

\section{Correlations for arbitrary barriers at quadratic irrational frequen- cies}

In this section we study correlations at quadratic irrational frequencies for arbitrary barriers. We begin with a summary from our previous paper [12]. We define a barrier in the following way:

Definition. A barrier $B$ consists of a finite set of intervals $\left[l_{i}, u_{i}\right], i=1,2, \ldots, n$ such that $0 \leq l_{1}<u_{1}<l_{2}<u_{2}<$ $\cdots<l_{n}<u_{n} \leq 1$. 
We restrict our attention to a subset of these objects and define the $\alpha$-barrier to be the barrier consisting of the single interval $[0, \alpha]$. This barrier is shown in Figure 12, along with its associated barrier function $\Phi_{\alpha}(\theta)$. However the general barrier $B$ is just finite product of barriers of this type and so we can focus on these without loss of generality due to the multiplicative nature of (2.18).
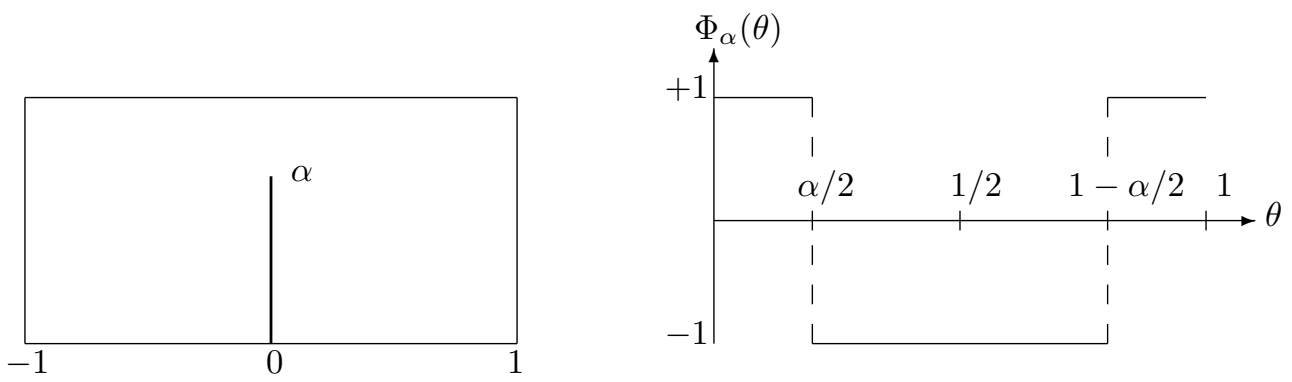

Figure 12: The $\alpha$-barrier and its associated barrier function $\Phi_{\alpha}(\theta)$.

Now as a result of Section 3 we see that periodic points of $F$ (as defined in (3.4)) correspond to periodic discontinuities of $Q_{n}$. Recall from (2.18) that the initial conditions for the recursion are $Q_{0}=1, Q_{1}=\Phi(-\omega x)$ and so with initial condition $\Phi_{\alpha}(-\omega x)$ as above we conclude that for periodic behaviour of the renormalization operator we must select $\alpha \in \mathbb{Q}(\omega)$. If $\alpha \notin \mathbb{Q}(\omega)$ then the functions $Q$ cannot be periodic as the locations of the discontinuities will not be periodic. This section is concerned with what happens when we select the barrier height alpha outside the field $\mathbb{Q}(\omega)$.

In [12] for the golden mean case $m=1$ we chose $\alpha=\sqrt{2}-1 \notin \mathbb{Q}(\omega)$. We then numerically approximated the integrals $C\left(G_{n}\right)$ giving the time series $\left(C\left(G_{n}\right)\right)$, and plotted the triple $\left(C\left(G_{n}\right), C\left(G_{n+1}\right), C\left(G_{n+2}\right)\right)$ which by the work of Takens [16] gives an embedding of the "attractor" as shown below in Figure 16a.

We have named this object "the star" for obvious reasons. The correlations are chaotic as the largest Lyapunov exponent for the associated time series is found to be positive in [12] using Rosenstein's method [15]. Now the question pertains as to what happens to this object as we increase $m$. In particular we study the cases $m=2,3,4$.

The same approach as in [12] has been adopted in this paper, and we have in each case $(m=2,3,4)$ calculated a positive largest Lyapunov exponent for the numerically approximated time series $\left(C\left(G_{n}\right)\right)$ using Rosenstein's method [15], thus indicating that the correlations are indeed chaotic.

We begin by analysing the two dimensional projections of the object i.e. the plots $C\left(G_{n+1}\right)$ versus $C\left(G_{n}\right)$ and $C\left(G_{n+2}\right)$ versus $C\left(G_{n}\right)$. As in [12] for the case of $m=1$, we have taken $\alpha \notin \mathbb{Q}(\omega)$. The plots of these projections of the surface are given in Figure 13.

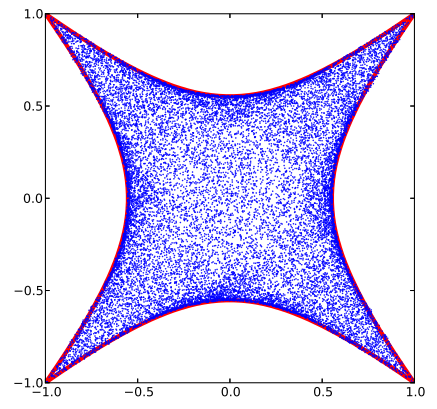

(a) $C\left(G_{n+1}\right)$ vs. $C\left(G_{n}\right)$

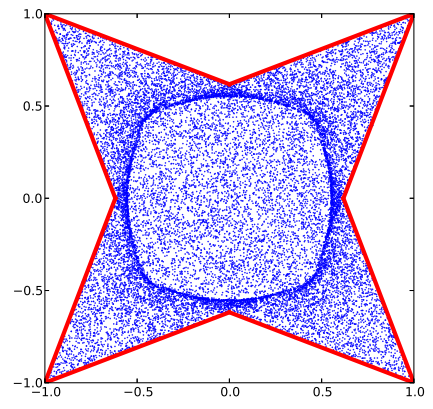

(b) $C\left(G_{n+2}\right)$ vs. $C\left(G_{n}\right)$

Figure 13: Chaotic correlations in the golden mean case. 
We observe that the boundary of Figure 13a consists of four symmetric hyperbolic curves crossing the axes at $1-1 / \sqrt{5}$, and in [12] we conjecture that left and right curves of the boundary (shown in red) are given by the hyperbola

$$
C\left(G_{n+1}\right)^{2}-\left(1-a^{2}\right) C\left(G_{n}\right)^{2}=a^{2},
$$

where $a=1-1 / \sqrt{5}$. In Figure 13b we observe that the boundary is piece-wise linear.

We will provide the same analysis now for the cases $m=2,3,4$. In Figures 14 and 15 we give the projections $C\left(G_{n+1}\right)$ and $C\left(G_{n+2}\right)$ versus $C\left(G_{n}\right)$ respectively.

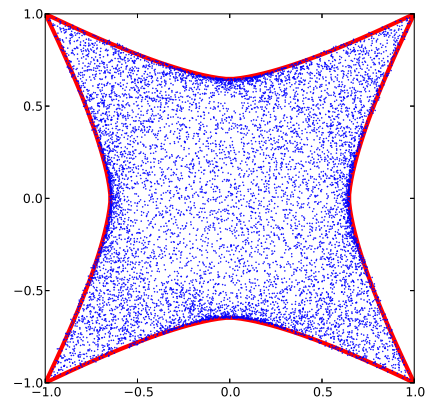

(a) $m=2$

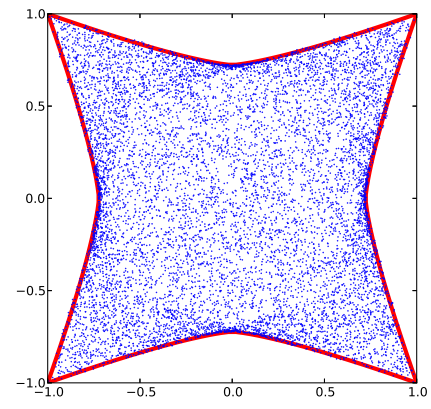

(b) $m=3$

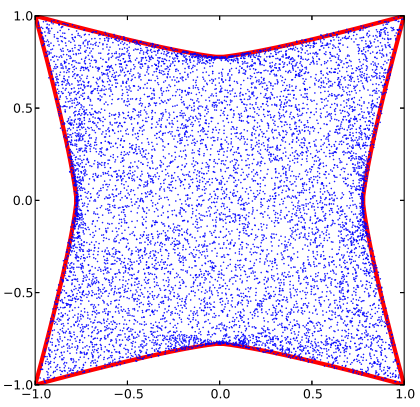

(c) $m=4$

Figure 14: Plot of $C\left(G_{n+1}\right)$ against $C\left(G_{n}\right)$ for $m=2,3,4$

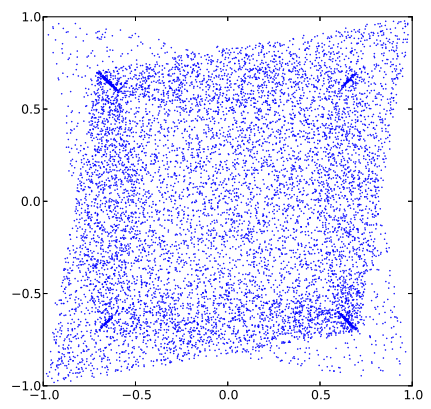

(a) $m=2$

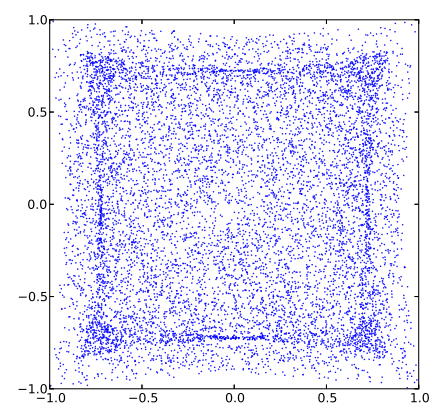

(b) $m=3$

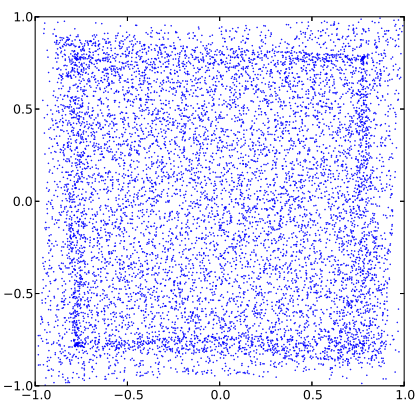

(c) $m=4$

Figure 15: Plot of $C\left(G_{n+2}\right)$ against $C\left(G_{n}\right)$ for $m=2,3,4$

In Figure 14 we see that as $m$ increases the topology of the set remains similar, with the correlations exploring an invariant set bounded by smooth curves. These curves are widening, crossing the axes now at the values $1-1 / \sqrt{m^{2}+4}$, the value calculated for the asymptotic peaks of the ACF for the half barrier earlier. The curves are also becoming more linear, and so we conjecture that as $m \rightarrow \infty$ the correlations will densely explore the unit square. We have also observed that the left and right portions of the boundary are given by the curves

$$
\left|C\left(G_{n+1}\right)\right|^{p}-\left(1-a^{p}\right)\left|C\left(G_{n}\right)\right|^{p}=a^{p}, \quad a=1-1 / \sqrt{m^{2}+4}, \quad p=(m+1) / m,
$$

the remaining curves follow by reflection along the diagonal, and this conjectured boundary is shown in red in each case. This tantalising observation gives us some hope that we may be able to shed some light on the nature of the invariant sets. Note that $a^{p} \rightarrow 1$ as $m \rightarrow \infty$ so the curves are tending to the constants $C\left(G_{n+i}\right)= \pm 1$ for $i=0,1$ (after reflections), in particular they are becoming the sides of the unit square. 
For Figure 15 we see that for $m=2$ the projection appears to be the intersection of two "planes", whereas for larger $m$ the structure becomes more complex, suggesting further intersections.

Finally, in Figure 16 we present the embed in three dimensions by plotting the triple $\left(C\left(G_{n}\right), C\left(G_{n+1}\right), C\left(G_{n+2}\right)\right)$. This reveals the presence of an invariant set or "attractor" embedded in this space.

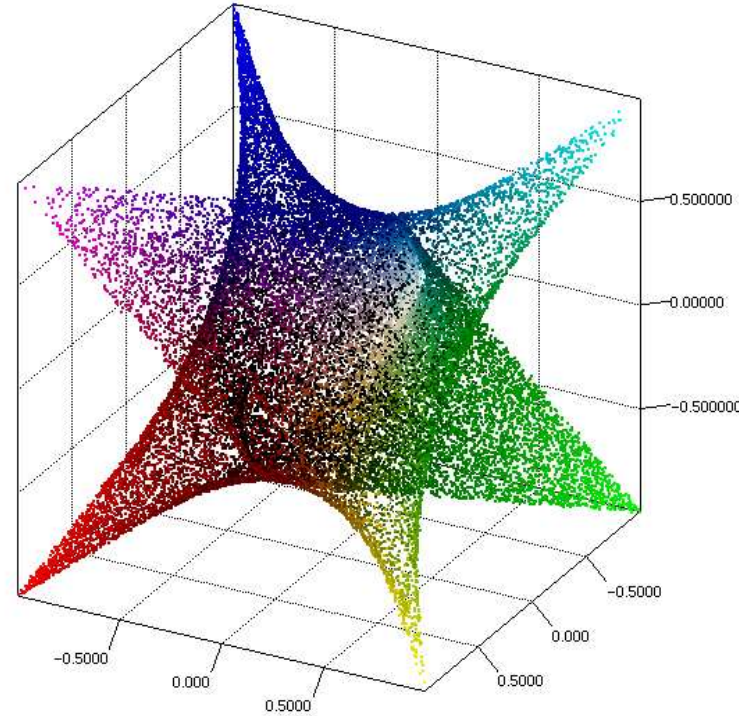

(a) $m=1$

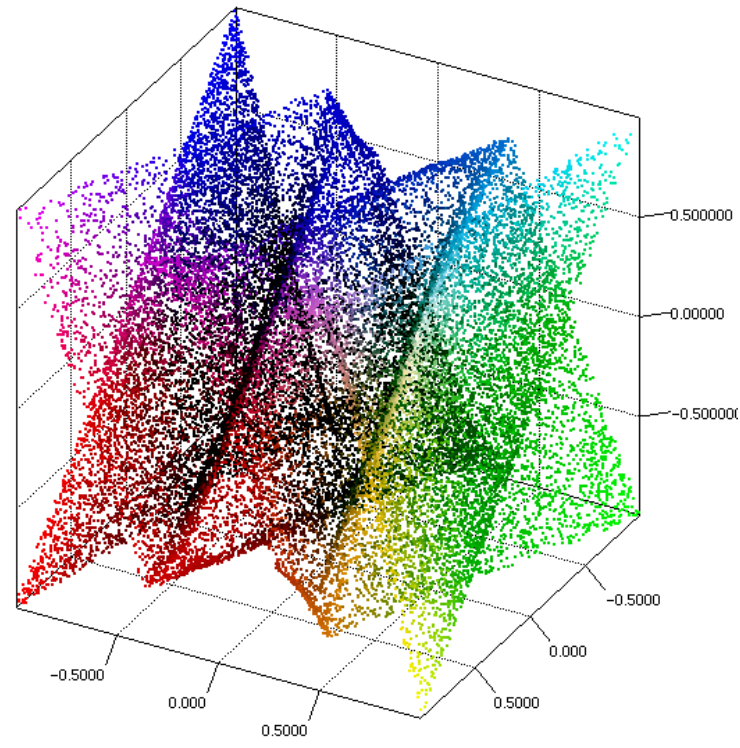

(c) $m=3$

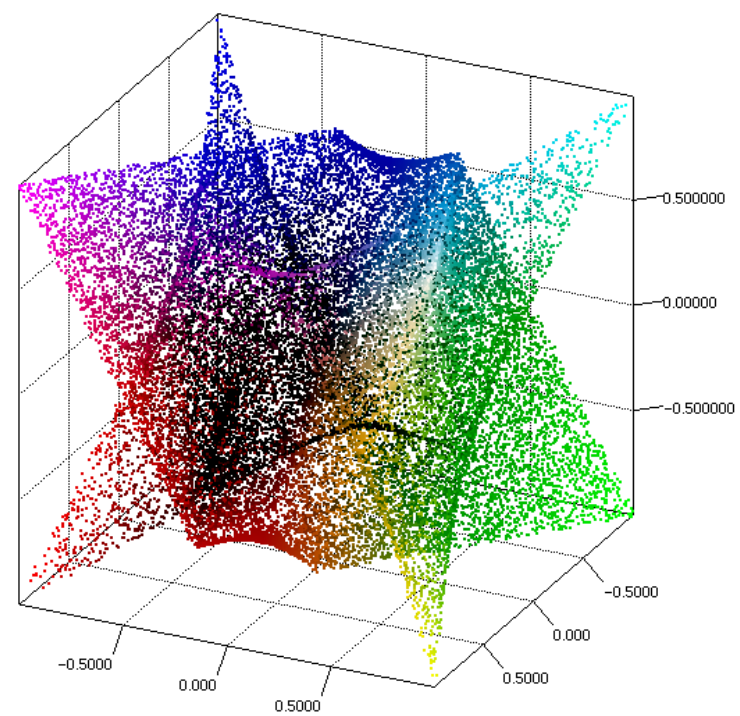

(b) $m=2$

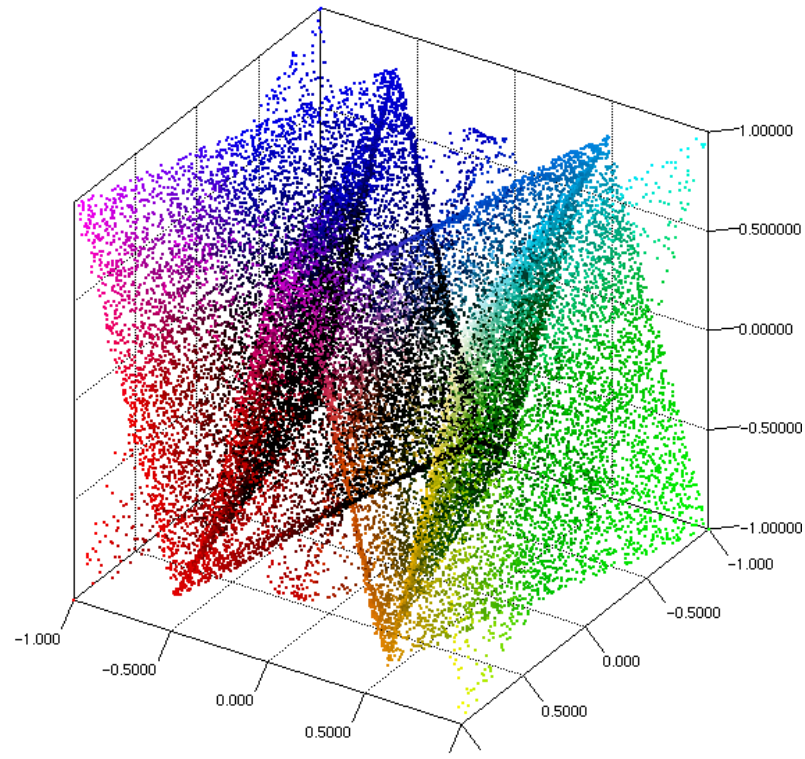

(d) $m=4$

Figure 16: Plot of the triple $\left(C\left(G_{n}\right), C\left(G_{n+1}\right), C\left(G_{n+2}\right)\right)$ for $m=2, \ldots, 4$, revealing in each case an invariant set embedded in three dimensional space representative of the underlying dynamics

Note that this "surface" is becoming more topologically complex as we increase $m$, and that we can see more and more repetition of internal structures. As a result of the symmetry in all the plots displayed (as before in [12]), 
and multiplicative decomposition of an arbitrary barrier into $\alpha$-barriers, the "attractor" obtained for an arbitrary barrier will be identical and is independent for $\alpha \notin \mathbb{Q}(\omega)$.

\section{Conclusion}

We have extended the study of the self-similarity and chaoticity of the autocorrelation function (ACF) of symmetric barrier billiards to a class of general quadratic irrational frequencies. The paper presents a renormalization analysis of the of the ACF for symmetric barrier billiards, much in the spirit of previous papers [1], [12] for the golden mean frequency.

This analysis has been heavily motivated by the work on quadratic irrational frequencies for a quasi-periodically forced two-level system in [10]. It depends on a piecewise function $F$, whose periodic orbits correspond to discontinuities of the recurrence (2.18) inside the fundamental interval. Numerical results for such frequencies first appeared in [13] when the authors examined the ACF for a strange non-chaotic attractor for the silver mean $(\omega=\sqrt{2}-1)$ frequency.

In summary we have shown that, for the half barrier, we must consider $m$ modulo four to get a complete understanding of the ACF which shows a self similar form at times $G_{n}$ (the generalised Fibonacci numbers as defined in equation (2.10)). However, the main features of the ACF are determined by the parity of $m$ : When $m$ is odd, we have peaks of magnitude $1-1 / \sqrt{m^{2}+4}$ at $G_{3 n}(n \geq 1)$ and these peaks alternate in sign; when $m$ is even we have peaks of the same magnitude at $G_{2 n}$.

Furthermore there is a discrepancy between the cases $m \equiv 0(\bmod 4)$ and $m \equiv 2(\bmod 4)$; in the former the peaks are all positive whereas in the latter they alternate in sign.

We have also extended our study in [12] in the same manner, revealing the persistent presence of invariant sets on which the (chaotic) correlations at characteristic times lie for increasing $m$. We see that the structure projected in three dimensions becomes topologically complex as shown in Figure 16, perhaps manifesting continual repetition of pre-existing structures.

These results should be readily extendible to quadratic frequencies with eventually periodic continued fraction expansion; however an analysis of general irrational frequencies will be highly challenging as the recurrence taking the role of (2.18) will change from step to step and depend on the entries in the continued faction expansion of the frequency, and hence we cannot expect self-similar behaviour. The related equations are not difficult to derive, but whether any meaningful information can be gained from them is currently unclear.

Of manifest interest is the structure and nature of the objects in Figure 16. Indeed, in [9] the authors analysed a generalised Harper equation for the golden mean flux, in particular giving a study of the renormalization strange set first reported in [5] called "the Ketoja-Satija Orchid". In doing so they provided a rigorous description of its structure and properties. They then extended their analysis to quadratic irrationals [8], giving rise to a "garden of orchids". The invariant sets shown in this paper are the equivalent of this for barrier billiards, and we hope to perform a similar analysis for this problem. Firstly for the golden mean case, the set which we name "the star" (Figure 16a), and then for quadratic irrationals giving rise to a "constellation of stars".

\section{References}

[1] J. R. Chapman and A. H. Osbaldestin, Self-similar correlations in a barrier billiard, Physica D 180 (2003), no. $1,71-91$.

[2] J Dalton and B D Mestel, Renormalization for the harper equation for quadratic irrationals, Journal of Mathematical Physics A 44 (2003), 4776-4783. 
[3] U. Feudel, A. Pikovsky, and A. Politi, Renormalization of correlations and spectra of a strange non-chaotic attractor, Journal of Physics A: Mathematical and General 29 (1996), no. 17, 5297-5311.

[4] U. Feudel, A. S. Pikovsky, and M. A. Zaks, Correlation properties of a quasiperiodically forced two-level system, Physical Review E 51 (1995), no. 3, 1762-1769.

[5] J. A. Ketoja and I. I. Satija, Self-similarity and localization, Physical Review Letters 75 (1995), no. 14, 27622765 .

[6] S P Kuznetsov, A S Pikovsky, and U Feudel, Birth of a strange nonchaotic attractor: A renormalization group analysis, Physical Review E 51 (1995), no. 3, R1629-R1632.

[7] B D Mestel and A H Osbaldestin, Periodic orbits of renormalisation for the correlations of strange nonchaotic attractors., Mathematical Physics Electronic Journal [electronic only] 6 (2000), no. 5, 27.

[8] __ A garden of orchids: a generalized harper equation at quadratic irrational frequencies, Journal of Physics A: Mathematical and General 37 (2004), no. 39, 9071-9086.

[9] _ Golden mean renormalization for a generalized Harper equation: The Ketoja-Satija orchid, Journal of mathematical physics 45 (2004), 5042-5075.

[10] _ Renormalization of correlations in a quasiperiodically forced two-level system: quadratic irrationals, Journal of Physics A: Mathematical and General 37 (2004), no. 38, 8969-8989.

[11] B D Mestel, A H Osbaldestin, and B Winn, Golden mean renormalization for the harper equation: the strong coupling fixed point, Journal of Mathematical Physics 41 (2000), 8304-8330.

[12] A H Osbaldestin and L N C Adamson, Chaotic correlations in barrier billiards with arbitrary barriers, Journal of Physics A: Mathematical and Theoretical 46 (2013), no. 24, 245101.

[13] A. S Pikovsky and U. Feudel, Correlations and spectra of strange nonchaotic attractors, Journal of Physics A: Mathematical and General 27 (1994), no. 15, 5209-5219.

[14] G. W. Riley, On spectral properties of skew products over irrational rotations, Journal of the London Mathematical Society 2 (1978), no. 1, 152-160.

[15] M. T. Rosenstein, J. J. Collins, and C. J. De Luca, A practical method for calculating largest lyapunov exponents from small data sets, Physica D: Nonlinear Phenomena 65 (1993), no. 1, 117-134.

[16] F. Takens, Detecting strange attractors in turbulence (lecture notes mathematics vol 898), Berlin: Springer (1981), 366-381.

[17] J. Wiersig, Singular continuous spectra in a pseudointegrable billiard, Physical Review E 62 (2000), no. 1, R21-R24. 